\title{
Resource Allocation for D2D Links in the FFR and SFR Aided Cellular Downlink
}

\author{
Shruti Gupta ${ }^{1}$, Suman Kumar ${ }^{2}$, R. Zhang ${ }^{1}$, S. Kalyani ${ }^{3}$, K. Giridhar ${ }^{3}$ and L. Hanzo ${ }^{1}$ Fellow, IEEE
}

\begin{abstract}
Device-to-Device (D2D) communication underlying cellular networks, allows direct transmission between two devices in each other's proximity that reuse the cellular resource blocks in an effort to increase the network capacity and spectrum efficiency. However, this imposes severe interference that degrades the system's performance. This problem may be circumvented by incorporating fractional frequency reuse (FFR) or soft frequency reuse (SFR) in OFDMA cellular networks. By carefully considering the downlink resource reuse of the D2D links, we propose beneficial frequency allocation schemes, when the macrocell has employed FFR or SFR as its frequency reuse technique. The performance of these schemes is quantified using both analytical and simulation results for characterising both the coverage probability and the capacity of D2D links under the proposed schemes that are benchmarked against the radical Unity Frequency Reuse (UFR) scheme. The impact of the D2D links on the coverage probability of macro-cellular users (CUs) is also quantified, revealing that the CUs performance is only modestly affected under the proposed frequency allocation schemes. Finally, we provide insights concerning the power control design in order to strike a beneficial trade-off between the energy consumption and the performance of D2D links.
\end{abstract}

\section{INTRODUCTION}

With the spread of mobile devices, tablets and mobile multimedia services, the amount of traffic conveyed by the cellular networks has been escalating. Hence the macrocell base stations (MBS) have to handle more traffic in order to meet the demand of high data rate services. Clearly, there is a need for enhancing the capacity of the cellular network in order to accommodate the deluge of multimedia traffic, which requires increasing the tele-traffic capacity, employing more MBSs etc. However, the radio resources available for cellular communications are limited and the employment of more MBSs is uneconomical. Therefore, further research is required for improving the capacity of cellular systems, whilst relying on the existing infrastructure. In the existing cellular networks, the data of all the cellular users (CUs) is relayed through the MBSs, even though the CUs may be closely located, which in turn increases both the delay as well as the traffic load imposed on the MBSs due to the high density of users. The solution to this problem is a promising new local

1. School of Electronics and Computer Science, University of Southampton, SO17 1BJ, United Kingdom.

Corresponding Author's Email: 1h@ecs.soton.ac.uk

2. Electrical Department, Indian Institute of Technology, Ropar

3. Electrical Department, Indian Institute of Technology, Madras

The financial support of the EPSRC projects EP/N004558/1, EP/N023862/1 and EP/L018659/1, as well as of the European Research Council's Advanced Fellow Grant under the Beam-Me-Up project and of the Royal Society's Wolfson Research Merit Award is gratefully acknowledged. ad-hoc networking technology, known as Device-to-Device (D2D) communication, which allows closely located devices to communicate directly by reusing the frequency band of the operational cellular network [1], [2].

However, the D2D links impose additional interference on the communication system. Explicitly, if a D2D link relies on utilising downlink (DL) resources, then the signals transmitted by the MBS to CUs may cause interference at the $\mathrm{D} 2 \mathrm{D}$ receivers, while the $\mathrm{D} 2 \mathrm{D}$ transmissions would degrade the DL channel quality of MBSs transmitting to the CUs. Furthermore, there exist interferences amongst the D2D links themselves, which reuse the same frequency bands. In order to maintain the Quality of Service (QoS) target both for the CUs and the D2D links, beneficial resource reuse schemes have been proposed for D2D communication, where the macrocell employed Unity Frequency Reuse (UFR) [3]-[8]. However, the interference imposed on the CUs can be significantly reduced by using Inter-Cell Interference Coordination (ICIC) schemes, such as, Fractional Frequency Reuse (FFR) and Soft Frequency Reuse (SFR). In FFR and SFR, the interference is eliminated by carefully coordinating the frequency bands used among the cells as well as by partitioning the total frequency band of each cell into a cell-centre region (CCR) and cell-edge region (CER) frequency-set. These frequency reuse techniques have been investigated in the literature in the context of different network models [9]-[13]. Xu et al. [9] derived the optimal radius to identify the CER and CCR users and quantified the attainable throughput. Gonzalez et al. proposed [10] to improve both the network capacity and the cell-edge performance for a dynamic SFR deployment relying on realistic irregularly shaped cells. Distributed antenna aided FFR was conceived by Zhang et al. for maximising both the cell-throughput and the coverage quality [11]. Kumar et al. [12] analysed the coverage probability of their proposed frequency allocation schemes for picocell users, when the macrocell employed FFR and SFR. As a further development [13], Jin et al. proposed the so-called spectrum swapping access strategy for a twin-layer network relying on FFR.

Furthermore, several resource allocation algorithms have been conceived for D2D communication in cellular networks using FFR [14]-[19]. In particular, a novel D2D-aware dynamic FFR algorithm was proposed in [14]. In [15], resource allocation was designed by $\mathrm{Wu}$ and Zhang for D2D communication in an FFR scenario by formulating it as binary integer optimization problem. Chae et al. [16] observed a significant improvement in the attainable cell throughput with 
the aid of their radio resource allocation scheme proposed for D2D links based on their specific location in the cell, when the cellular network was relying on FFR. Kim et al. [17] proposed a resource allocation algorithm for eliminating the interferences imposed by the D2D links, while the spectral efficiency of D2D communication achieved in FFR-aided OFDMA cellular systems was analytically investigated by Zhu and Wang [18].

\section{(a) FFR}

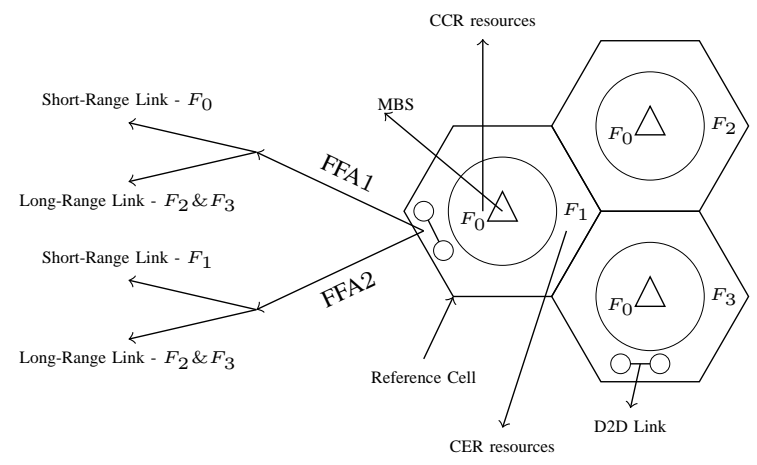

(b) SFR

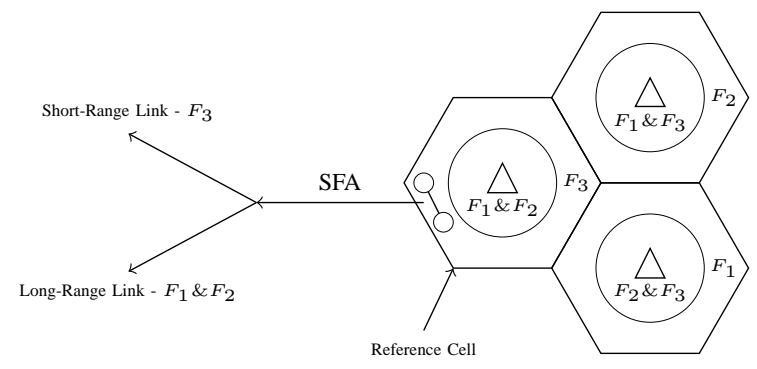

Figure 1. Frequency resource allocation in macrocell and D2D links in FFR and SFR

Against this background, we consider a cellular network employing FFR or SFR in conjunction with D2D communication relying on downlink resource reuse. The devices of D2D links are battery-operated, hence it is essential to save energy at these devices using power control in order to prevent the batteries from running out as well as for reducing the interference imposed on the CUs. However, at the same time it is necessary to ensure that the power control of the D2D links should not significantly degrade the performance of these links. The major contributions of this treatise are as follows:

1) We have proposed a pair of fractional frequency allocation schemes (FFA1 and FFA2) for D2D links, when the macrocell relies on an FFR scheme, where the D2D links are classified into two categories based on a signal-tointerference-ratio (SIR) threshold $S_{d}$, namely the shortrange (SR) D2D links and the long-range (LR) D2D links. The frequency allocation schemes are formulated as ${ }^{1}$ :

a) Fractional Frequency Allocation 1 (FFA1): Consider the macrocell 0 of Fig. 2 as the reference cell, where $F_{0}$ is CCR frequency and $F_{1}$ is CER frequency of this macrocell while CER frequency of the neighbouring cells is $F_{2}$ and $F_{3}$. In this scheme, we allocate the CCR frequency $\left(F_{0}\right)$ of the reference macrocell to the short-range D2D links and the CER frequency ( $F_{2}$ and $F_{3}$ ) of the other macrocells to the long-range D2D links, as shown in Fig. 1(a).

b) Fractional Frequency Allocation 2 (FFA2): Similar to FFA1, considering the reference macrocell as macrocell 0 of Fig. 2, in this scheme, we allocate the CER frequency $\left(F_{1}\right)$ of the reference macrocell to the SR D2D links and the CER frequency $\left(F_{2}\right.$ and $\left.F_{3}\right)$ of the other macrocells to the LR D2D links, as shown in Fig. 1(a).

2) We have also proposed a frequency allocation scheme for the D2D links, when the macrocell has employed a SFR scheme. The scheme is specified as follows:

a) Soft Frequency Allocation (SFA): Again considering the reference macrocell as macrocell 0 of Fig. $2, F_{3}$ is the CER frequency, while $F_{1}$ and $F_{2}$ represent the CCR frequency. In this scheme, we allocate the CER frequency $\left(F_{3}\right)$ to the SR D2D links and the CCR frequency $\left(F_{1}\right.$ and $F_{2}$ ) to the LR D2D links, as shown in Fig. 1(b).

All the proposed schemes defined above are motivated by the FFR scheme itself, where the CCR users (or SR D2D links in the proposed schemes) experience more interference than the CER users (or LR D2D links). We then analytically derived both the coverage probability and the capacity for the D2D links corresponding to all the proposed schemes. Simulation results are provided for validating our analytical results. Then our proposed schemes are compared to the benchmark scheme, where the MBS relies on UFR. The results reveal that the proposed schemes significantly outperform the benchmark scheme. The impact of the D2D links on the CUs is also quantified. Moreover, we have provided an intuition concerning the selection process of the power control factor in order to strike a compelling trade-off between the energy consumption and the performance of the D2D links.

The rest of the paper is organized as follows. In Section II, our system model is presented, which is followed by the analytical derivation of both the coverage probability and the capacity of the D2D links for our proposed frequency allocation schemes in Section III. Our performance results are discussed in Section IV, whilst our conclusions are offered in Section V.

\footnotetext{
${ }^{1}$ Note that in Fig. 1, the different frequency reuse zones are shown only for illustration. However, the specific frequency resources used by the CUs/D2D links depend on their received SIR, rather than on the geographic location of the CUs/D2D links.
} 


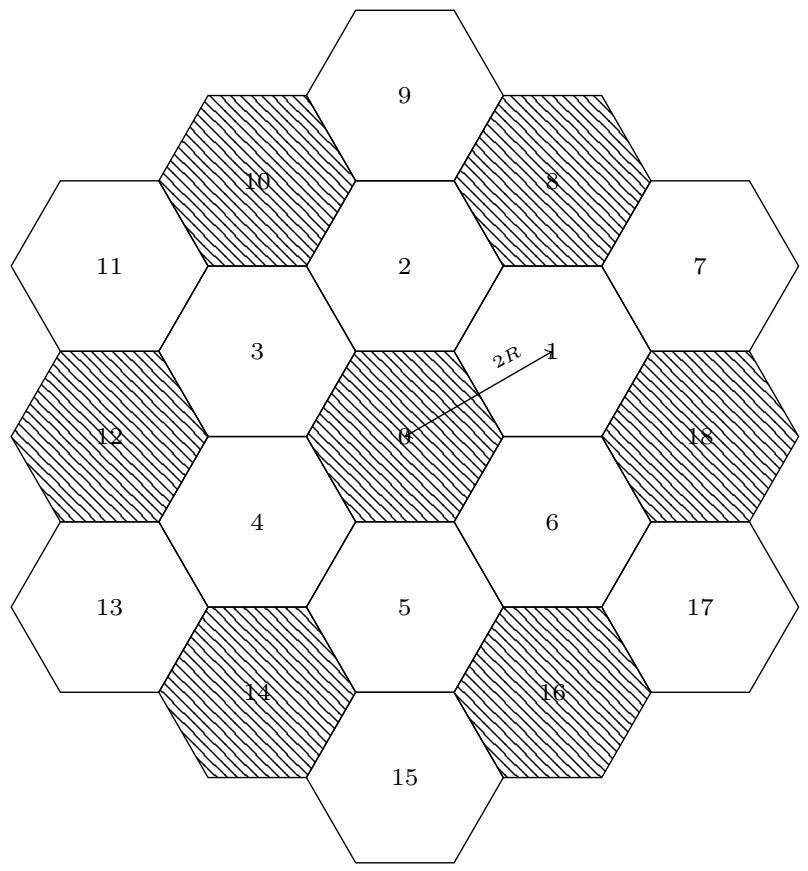

Figure 2. Hexagonal macrocell structure. The interference imposed by a UFR system on cell 0 is contributed by all the 18 neighbouring cells, while in a frequency reuse $\frac{1}{3}$ system it is contributed only by the shaded cells.

\section{SYSTEM MODEL}

We considered the cellular network shown in Fig. 2, supporting both orthogonal downlink cellular users (CUs) and D2D links where each macrocell is approximated by a circle of radius $R_{c}$. Considering the downlink of a cellular system, we introduced multiple D2D links that reuse the downlink resources of multiple CUs, where each CU occupies a dedicated resource block (RB).We assume that each CU's $\mathrm{RB}$ can be reused by at most one D2D link. This means that a single D2D link can reuse the resource blocks of several CUs, but one of the CU's resource blocks can be reused by only one D2D link at a time. In essence, this may be interpreted as a mapping of one D2D link to multiple resource blocks of different CUs. A similar D2D related constraint was imposed on the cellular user's RB in [20] and [21]. All possible transmission channels in the network are considered to be independent and identically Rayleigh distributed throughout this treatise. The D2D communication is incorporated as a complement to the underlying cellular communication and thus the CUs generally have a higher priority than the D2D links in a cell. The BS maintains reliable connection with the CUs under the power budget of $P_{c}$, while the D2D link reuses the randomly matched RB of the CU under the power budget $P_{d}^{\max }$ along with a power control factor of $\epsilon$. We assume that all the D2D links use a distance-dependent proportion of the total power [22]. In other words, the transmit power $P_{d}$ of the D2D link is formulated as follows:

$$
P_{d}(r)=P_{d}^{\max }\left(\frac{r}{R_{2}}\right)^{\alpha \epsilon}, \quad \forall r \in\left[R_{1}, R_{2}\right]
$$

where $r$ is the distance and $\alpha$ is the path-loss exponent between the D2D transmitter (Tx) and receiver $(\mathrm{Rx})$. The minimum and maximum distance of the D2D link are denoted by $R_{1}$ and $R_{2}$, respectively. The power control factor of $\epsilon \in[0,1]$ controls the power transmitted by the D2D Tx. A lower power control factor allows the D2D Tx to transmit at higher power, which might result in higher coverage quality and higher capacity for the D2D link. On the other hand, a higher power control factor reduces the amount of transmit power used by the D2D link. It is apparent from Eq. (1) that at $\epsilon=0$ the transmit power of all the D2D links present in the macrocell is $P_{d}^{\max }$, while at $\epsilon=1$ the transmit power would be at its minimum. Furthermore, when the D2D Tx and Rx have the maximum distance of $r=R_{2}$, the transmit power for that D2D link would be $P_{d}^{\max }$, regardless of the value of $\epsilon$.

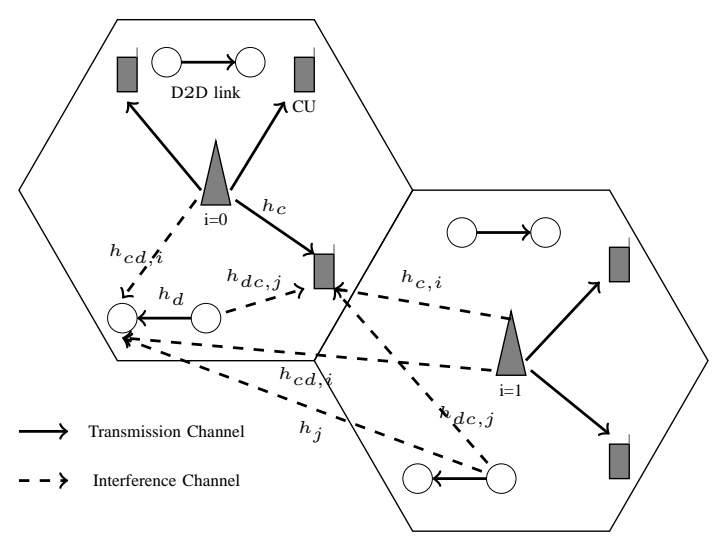

Figure 3. Illustration of the interfering links for two adjacent macrocells, when a D2D link is superimposed on a CU's RB.

When the MBS relies on UFR, the SIR at the D2D Rx, which is at a distance of $l$ from the reference MBS and with a separation of $r$ from the $\mathrm{D} 2 \mathrm{D} \mathrm{Tx}$, is given by:

$$
\begin{aligned}
\gamma_{U}(l, r) & =\frac{P_{d}(r) h_{d} r^{-\alpha}}{I_{c d}+I_{d}}, \\
I_{c d} & =\sum_{i \in \phi} P_{c} h_{c d, i} l_{i}^{-\alpha}, \\
I_{d} & =\sum_{j \in \psi \backslash\{0\}} P_{d}\left(r_{j}\right) h_{j} d_{j}^{-\alpha} .
\end{aligned}
$$

Here $P_{d}(r)$ and $P_{c}$ denote the transmit power of the D2D Tx and of the MBS, respectively. Furthermore, $I_{c d}$ is the interference experienced by the D2D Rx due to the downlink cellular communication, i.e. the interference caused by the MBSs transmitting on the same frequency band in the network, while $I_{d}$ is the interference imposed by D2D links in other macrocells that reuse the same frequency band. The fadinginduced attenuation experienced by the channel between the D2D transmitter and receiver is $h_{d}$, while that of the channel spanning from the $i^{t h}$ MBS to the D2D Rx is $h_{c d, i}$ and that of the $j^{t h} \mathrm{D} 2 \mathrm{D} \mathrm{Tx}$ in other macrocells to the D2D Rx is $h_{j}$. For the sake of better understanding, these channel fading gains for two adjacent macrocells are shown in Fig. 3, which can be 
easily extended to our network model of Fig. 2. Furthermore, $\phi$ is the set of all macrocells present in the cellular network of Fig. 2, while $\psi$ is the set of all the D2D links in other macrocells, operating on the same frequency set. Similarly, the SIR of CUs at a distance $d_{c}$ from the MBS of the macrocell which is using UFR, can thus be written as:

$$
\begin{aligned}
\gamma_{c}\left(d_{c}\right) & =\frac{P_{c} h_{c} d_{c}^{-\alpha}}{I_{c}+I_{d c}}, \\
I_{c} & =\sum_{i \in \phi \backslash\{0\}} P_{c} h_{c, i} d_{c, i}^{-\alpha}, \\
I_{d c} & =\sum_{j \in \psi} P_{d}\left(r_{j}\right) h_{d c, j} l_{d c, j}^{-\alpha},
\end{aligned}
$$

where $h_{c}$ denotes the fading gain of the MBS to CU link, $h_{c, i}$ is fading gain experienced from the $i^{\text {th }}$ MBS using the same frequency band to the $\mathrm{CU}$ and $h_{d c, j}$ is the fading gain of the $j^{\text {th }}$ D2D Tx to the CU link operating in the same frequency band, as shown in Fig. 3. Here $I_{c}$ represents the interference imposed by the MBSs on the CU using the same frequency band, while $I_{d c}$ is the interference experienced by the $\mathrm{CU}$ due to the superimposed D2D links.

We consider two different frequency reuse schemes, namely the FFR and SFR regimes. In the FFR scenario, the total available bandwidth is divided into four orthogonal frequency bands, obeying $F=F_{0}+F_{1}+F_{2}+F_{3}$. More particularly, the frequency band $F_{0}$ is common to all the macrocells for the CCR region of the network, while $F_{i}, i \in 1,2,3$ is assigned to the users in the CER of the three adjacent macrocells, as shown in Fig. 1(a). The classification of CUs roaming in the CCR and CER of the macrocell is determined on the basis of their SIR and the predefined threshold SIR $S_{c}$. Now, in order to allocate an adequate frequency band to $\mathrm{CU}$, we have to categorise them into CCR and CER users. The users that have an SIR higher than the predefined threshold SIR $\left(\gamma_{c}>S_{c}\right)$ constitute the CCR users, while the ones with an SIR lower than the threshold $\left(\gamma_{c} \leq S_{c}\right)$ are the CER users.

On the other hand, when considering SFR, the total available bandwidth is partitioned into three equal orthogonal frequency bands according to $F=F_{1}+F_{2}+F_{3}$, where one of the frequency bands $F_{i}, i \in 1,2,3$ is used in the CER of the macrocell ensuring that it is orthogonal to the neighbouring CER of the adjacent macrocells, while the remaining twothirds of the frequency band is reserved for the CCR of the macrocell, as depicted in Fig. 1(b). Moreover, the MBS would transmit at power $P_{m}$ in the CCR and at the power of $\beta P_{m}$ in the CER of the macrocell ${ }^{2}$.

\section{Coverage AND CAPACITY}

The coverage probability is defined as a probability of successful communication between the source and destination. In other words, the coverage probability of the D2D link is obtained as the probability of the D2D links possessing an SIR

$$
{ }^{2} P_{m}=\frac{P_{c}}{\beta}, \beta \geq 1
$$

higher than the target SIR $(T)^{3}$. It is affected by the distance $r$ between the D2D Tx and the D2D Rx, by the transmit power $P_{d}(r)$ of the D2D link as well as the interference $I_{c d}$ experienced at the D2D Rx due to the cellular communication and the interference $I_{d}$ owing to the D2D communication operating in the same frequency band. We would first derive the coverage probability of the D2D links, when the MBSs use UFR.

Lemma 1. The coverage probability of the D2D links, when the MBSs use UFR is given by Eq. (4) at the top of the next page.

Proof. See Appendix A for the proof.

\section{A. Fractional Frequency Reuse}

In UFR, the users roaming close to the MBS experience a lower co-channel interference than those, who are far from the MBS. However, the FFR relies on a combination of the frequency reuse factor of 1 and that of $\frac{1}{3}$, where the cell-centre users occupy a band having a reuse factor of 1 and the celledge users associated with a reuse factor of $\frac{1}{3}$. This implies that there is a reduction in the interference afflicted upon the cell-edge users due to the neighbouring cells. Let us first propose a pair of different frequency allocation schemes for the D2D links, when the MBSs use FFR and then derive the coverage probability of each of the proposed scheme. In order to define these two schemes, recall that we have classified the D2D link as SR D2D links and LR D2D links, based on the predefined threshold SIR $\left(S_{d}\right)$. The SR D2D link is defined as the link that has an SIR higher than the threshold SIR $\left(\gamma_{U}>S_{d}\right)$, while the LR D2D link has an SIR lower than the threshold SIR threshold $\left(\gamma_{U} \leq S_{d}\right)$. It is important to note that the SIR of the D2D link is calculated by assuming that the D2D link experiences interference from all the macrocells as well as from all the other D2D links that are present in the other macrocells and using the same frequency. Let us now describe our two frequency allocation schemes defined for the D2D links as follows:

1) Fractional Frequency Allocation 1 (FFA1): In this scheme, we allocate the CCR frequency of the reference macrocell to the SR D2D link and the CER frequency of the other macrocells to the LR D2D link. Upon considering the macrocell 0 of Fig. 2 as the reference cell, $F_{0}$ is the CCR frequency, while the CER frequency of the neighbouring cells is $F_{2}$ and $F_{3}$. Therefore, in FFA1, the SR D2D links would reuse $F_{0}$, while the LR D2D links would reuse $F_{2}$ and $F_{3}$.

2) Fractional Frequency Allocation 2 (FFA2): In this scheme, we allocate the CER frequency of the reference macrocell to the SR D2D link and the CER frequency of the other macrocells to the LR D2D link. Similar to FFA1, the reference macrocell is cell 0 of Fig. 2, where

\footnotetext{
${ }^{3}$ The target SIR for all the D2D links is considered to be the same for the analytical derivation of their coverage probability.
} 


$$
C P=\int_{0}^{R_{c}} \frac{R_{2}}{R_{2}-R_{1}} 2 F_{1}\left[\frac{1}{\alpha-\alpha \epsilon}, \theta(l), 1+\frac{1}{\alpha-\alpha \epsilon}, \frac{-\lambda(l) R_{2}^{\alpha} T}{P_{d}^{\text {max }}}\right]-\frac{R_{1}}{R_{2}-R_{1}}{ }_{2} F_{1}\left[\frac{1}{\alpha-\alpha \epsilon}, \theta(l), 1+\frac{1}{\alpha-\alpha \epsilon}, \frac{-\lambda(l) R_{1}^{\alpha-\alpha \epsilon} R_{2}^{\alpha \epsilon} T}{P_{d}^{\max }}\right] \frac{2 l}{R_{c}^{2}} \mathrm{~d} l .
$$

the SR D2D links would reuse $F_{1}$, which is the CER frequency of the reference cell and the LR D2D links would reuse $F_{2}$ and $F_{3}$.

The motivation behind this definition for the pair of schemes is the FFR scheme itself. In FFR, the cell-edge users (that have a low SIR) are assigned the frequency of $F_{i}, i \in 1,2,3$, which results in a reduced co-channel interference. Correspondingly, the D2D links that have a high SIR are assigned $F_{0}$ (or $F_{1}$ ), while the D2D links having a low SIR are assigned $F_{2}$ or $F_{3}$ in FFA1 (or FFA2) for the sake of reducing the co-channel interference. In other words, initially we try to serve the D2D link at $F_{0}$ (or $F_{1}$ ), i.e. we find the SIR based on $F_{0}$ (or $F_{1}$ ). However, the D2D link that experiences a high level of interference, i.e. whose SIR is low, is allocated a new frequency from the set of frequencies $F_{2}$ or $F_{3}$, which are not contaminated by the strong interference from reference MBS as shown in Fig. 1. The coverage probability of the D2D links when the MBSs use UFR was given by Eq. (26) of Appendix $A$ and we would now extend the same result to the proposed schemes.

Lemma 2. The coverage probability of a typical D2D link in the network of Fig. 2 using FFA1 is given by

$$
\begin{gathered}
C P_{F F A 1}=\int_{0}^{R_{c}} C P\left(l, \max \left\{T, S_{d}\right\}\right)+ \\
\hat{C P}(l, T)\left[1-C P\left(l, S_{d}\right)\right] f_{L}(l) d l,
\end{gathered}
$$

where $C P\left(l, \max \left\{T, S_{d}\right\}\right)$ and $C P\left(l, S_{d}\right)$ are defined in Eq. (25) from Appendix A. Similar to $C P(l, T)$ which is defined for the frequency $F_{0}, \hat{C P}(l, T)$ can be derived for frequency $F_{2}$ and $F_{3}$.

Proof. See Appendix B for the proof.

Similarly, the coverage probability of a D2D link using FFA2 in the network of Fig. 2 is given as follows:

$$
\begin{array}{r}
C P_{F F A 2}(l, r)=P\left[\tilde{\gamma}_{U}(l, r)>\max \left\{T, S_{d}\right\}\right]+ \\
P\left[\hat{\gamma}_{U}(l, r)>T\right] P\left[\tilde{\gamma}_{U}(l, r)<S_{d}\right] .
\end{array}
$$

It is important to note that in FFA2, the SR D2D link reuses the CER frequency band for its communication process and since the transmission channels are independent of each other, the D2D links experience different fading gains as well as interference, which is characterised by the SIR denoted by $\tilde{\gamma}_{U}(l, r)$. However, for the LR D2D links in FFA2, the frequency band reused is the same as that reused by the LR D2D links in FFA1 and hence the SIR is denoted by $\hat{\gamma}_{U}(l, r)$ in the above expression. Using the expression of coverage probability of the UFR defined in Eq. (26) we can write the final coverage probability expression of typical D2D links in the FFA2 scenario as:

$$
\begin{aligned}
& C P_{F F A 2}=\int_{0}^{R_{c}} \tilde{C P}\left(l, \max \left\{T, S_{d}\right\}\right)+ \\
& \hat{C P}(l, T)\left[1-\tilde{C P}\left(l, S_{d}\right)\right] f_{L}(l) \mathrm{d} l,
\end{aligned}
$$

where similar to $C P(l, T), \tilde{C P}(l, T)$ can be derived for the frequency $F_{1}$.

\section{B. Soft Frequency Reuse}

The macrocells relying on SFR techniques use one-third of the band allocated for the cell-edge users, which is set to be different from the neighbouring cells in order to avoid any interference, while the remaining two-thirds of the band is used for the cell-centre users. The MBS imposes power control for transmitting at the power of $P_{m}$ for the CCR users, while at $\beta P_{m}$ for the CER users. In this subsection, using the previous definitions of SR and LR D2D links, we propose a frequency allocation scheme for the D2D links, which is defined as follows:

1) Soft Frequency Allocation (SFA): In this scheme, we allocate the CER frequency to the SR D2D link and CCR frequency to the LR D2D link. Let us consider cell 0 of Fig. 2 as the reference macrocell, where the CCR and CER frequency bands are $F_{3}$ and $F_{1}, F_{2}$ respectively. According to the definition of this scheme, the SR D2D links are allocated $F_{3}$, while the LR D2D links reuse $F_{1}$ and $F_{2}$.

Similar to the previous derivation of the coverage probability for the FFR technique, we now continue by deriving the coverage probability for the proposed scheme, when network relies on SFR. We now derive the coverage probability of SFA.

Lemma 3. The coverage probability of the D2D link in SFA can be expressed as

$$
\begin{aligned}
& C P_{S F A}=\int_{0}^{R_{c}} C P_{S}\left(l, \max \left\{T, S_{d}\right\}\right)+ \\
& \hat{C} P_{S}(l, T)\left[1-C P_{S}\left(l, S_{d}\right)\right] f_{L}(l) d l,
\end{aligned}
$$

where similar to $C P(l, T)$ given in Eq. (25) from appendix $A$, $C P_{S}\left(l, \max \left\{T, S_{d}\right\}\right)$ and $\hat{C P}{ }_{S}(l, T)$ can be derived for the $C C R$ frequency and CER frequency.

Proof. See Appendix C for the proof. 


\section{Capacity}

In this subsection, we derive the capacity of all the proposed schemes as well as of the benchmark scheme. We commence with the benchmark scheme. The capacity is given by [12], [23],

$$
\begin{aligned}
C & =E[\ln (1+S I R)] \\
& =\int_{t>0} P[\ln (1+S I R)>t] \mathrm{d} t \\
& =\int_{t>0} P\left[S I R>e^{t}-1\right] \mathrm{d} t .
\end{aligned}
$$

Thus, the capacity is equivalent to the coverage probability evaluated at $T=e^{t}-1$ and then integrated over $t$. The capacity of a D2D link, which is at a distance of $l$ from the MBS is given by Eq. (10) (see next page). By averaging over the distance from the MBS and the distance between the D2D link, the capacity of a typical D2D link is given by

$$
\begin{array}{r}
C=\int_{0}^{R_{c}} \int_{R_{1}}^{R_{2}}{ }_{2} F_{1}\left(1,1 ; \theta(l)+1 ; 1-\frac{P_{d}^{\max }}{\lambda(l) r^{\alpha(1-\epsilon)} R_{2}^{\alpha \epsilon}}\right) \\
\frac{P_{d}^{\max }}{\theta(l) \lambda(l) r^{\alpha(1-\epsilon)} R_{2}^{\alpha \epsilon}} \frac{1}{R_{2}-R_{1}} \mathrm{~d} r \frac{2 l}{R_{c}^{2}} \mathrm{~d} l
\end{array}
$$

Now, we derive the capacity of the D2D link for the proposed FFA1 scheme. The capacity of an SR D2D link is given by

$$
\begin{gathered}
C_{S}(l, r)=\int_{t=0}^{\infty} \frac{P\left[\gamma_{U}(l, r)>\max \left\{e^{t}-1, S_{d}\right\}\right]}{P\left[\gamma_{U}(l, r)>S_{d}\right]} \mathrm{d} t \\
=\int_{t=0}^{\ln \left(1+S_{d}\right)} \frac{P\left[\gamma_{U}(l, r)>S_{d}\right]}{P\left[\gamma_{U}(l, r)>S_{d}\right]} \mathrm{d} t+ \\
\int_{\ln \left(1+S_{d}\right)}^{\infty} \frac{P\left[\gamma_{U}(l, r)>e^{t}-1\right]}{P\left[\gamma_{U}(l, r)>S_{d}\right]} \mathrm{d} t \\
C_{S}(l, r)=\ln \left(1+S_{d}\right)+P_{d}^{\max }{ }^{\infty}+\int_{t=\ln \left(1+S_{d}\right)}^{\infty}\left(\frac{1}{\lambda(l)\left(e^{t}-1\right) r^{\alpha(1-\epsilon)} R_{2}^{\alpha \epsilon}+P_{d}^{\max }}\right)^{\theta(l)} \mathrm{d} t
\end{gathered}
$$

After simplification, we arrive at,

$$
\begin{aligned}
& C_{S}(l, r)=\ln \left(1+S_{d}\right)+ \\
& \quad \frac{{ }_{2} F_{1}\left[\theta(l), \theta(l) ; \theta(l)+1 ;\left(1+S_{d}\right)^{-1}\left(1-\frac{P_{d}^{\max }}{\lambda(l) r^{\alpha(1-\epsilon)} R_{2}^{\alpha \epsilon}}\right)\right]\left(P_{d}^{\max }\right)^{\theta(l)}}{P\left[\gamma_{U}(l, r)>S_{d}\right]\left(\left(1+S_{d}\right) \lambda(l) r^{\alpha(1-\epsilon)} R_{2}^{\alpha \epsilon}\right)^{\theta(l)}}
\end{aligned}
$$

Similarly, the capacity of a LR link is given by

$$
\begin{aligned}
C_{L}(l, r) & ={ }_{2} F_{1}\left(1,1 ; \hat{\theta}(l)+1 ; 1-\frac{P_{d}^{\max }}{\hat{\lambda}(l) r^{\alpha(1-\epsilon)} R_{2}^{\alpha \epsilon}}\right) \times \\
& \frac{P_{d}^{\max }}{\hat{\theta}(l) \lambda(l) r^{\alpha(1-\epsilon)} R_{2}^{\alpha \epsilon}}
\end{aligned}
$$

Similar to $\theta(l)$ and $\lambda(l)$ which are given in Eq. (21) and are defined for a frequency reuse $1, \hat{\theta}(l)$ and $\hat{\lambda}(l)$ can be derived for a frequency reuse of $\frac{1}{3}$. Then the capacity of a typical $\mathrm{D} 2 \mathrm{D}$ link is given by

$$
\begin{aligned}
& C_{F F A 1}=\int_{0}^{R_{c}} \int_{R_{1}}^{R_{2}} C_{S}(l, r) P\left[\gamma_{U}(l, r)>S_{d}\right]+ \\
& \frac{2}{3} C_{L}(l, r) P\left[\gamma_{U}(l, r)<S_{d}\right] \frac{1}{R_{2}-R_{1}} \mathrm{~d} r \frac{2 l}{R_{c}^{2}} \mathrm{~d} l .
\end{aligned}
$$

The first term in Eq. (15) corresponds to the capacity of an SR D2D link that reuses $F_{0}$, whereas the second term denotes the capacity of the LR D2D link that reuses $F_{2}$ and $F_{3}$. Here the factor $\frac{2}{3}$ weights the second term due to the fact that among all the cell-edge sub-bands $\left(F_{1}, F_{2}, F_{3}\right)$, only $F_{2}$ and $F_{3}$ are used by the LR link in FFA1. The capacity of the other proposed schemes such as FFA2 and SFA can be derived using the process followed for FFA1. See Appendix $\mathrm{D}$ for the expressions of the capacity for the other schemes.

\section{Simulation Results}

In this section, we study the performance of the proposed schemes benchmarked against the scheme, when the MBS relies on UFR. In the scenario when the MBSs employ UFR, the frequency is reused according to a reuse factor of 1 and hence there is no need for any further classification into CCR and CER users, since all CUs will be using the same frequency band. Furthermore, a random frequency allocation scheme is assumed for the D2D links, which means that a random D2D$\mathrm{CU}$ association is considered for the resource reuse in the benchmark scenario. Furthermore, we analyse the impact of the proposed schemes on the CU coverage probability along with that of our benchmark scheme. We have considered the network of 19 hexagonal cells seen in Fig. 2, where each macrocell is approximated by a circle of radius $R_{c}=1000 \mathrm{~m}$ (except for Fig. 5) for ease of exposition. Each macrocell is a hybrid cell consisting of 100 CUs and 100 D2D links, where the MBS is located at the centre of the macrocell, while the CUs and the D2D links relying on downlink resources are distributed uniformly in the cell. Furthermore, all resource blocks are uniformly shared among the users and D2D links. In other words, if there are $K$ users or D2D links and $R$ resource blocks, then each user or each D2D link is assigned $\frac{R}{K}$ resource blocks. Furthermore, for each user we compute the SIR, which is used for classifying the users into the CCR and CER. Note that the SIR is evaluated based on the CCR region. In other words, we first assume that all the users are in the CCR region and then compute the SIR. Furthermore, we compare the SIR to $S_{c}$ and if the CU's SIR is higher than $S_{c}$, then the user will continue to rely on the same sub-band and will assumed to be a CCR user. By contrast, if the CU's SIR is lower than $S_{c}$, the user is assumed to be a CER user and hence will camp on new sub-band. Similarly, in a D2D link scenario, we first evaluate the SIR of the D2D user assuming that the D2D link is an SR D2D link and then compare the SIR to $S_{d}$. Now, if the D2D link's SIR is higher than $S_{d}$, the D2D link is assumed to be an SR link and will continue to use the same sub-band. By contrast, if the SIR is lower than 


$$
C(l, r)=\int_{t=0}^{\infty}\left(\frac{P_{d}^{\max }}{\lambda(l) r^{\alpha(1-\epsilon)} R_{2}^{\alpha \epsilon}+P_{d}^{\max }}\right)^{\theta(l)} \mathrm{d} t={ }_{2} F_{1}\left(1,1 ; \theta(l)+1 ; 1-\frac{P_{d}^{\max }}{\lambda(l)\left(e^{t}-1\right) r^{\alpha(1-\epsilon)} R_{2}^{\alpha \epsilon}}\right) \frac{P_{d}^{\max }}{\theta(l) \lambda(l) r^{\alpha(1-\epsilon)} R_{2}^{\alpha \epsilon}} .
$$

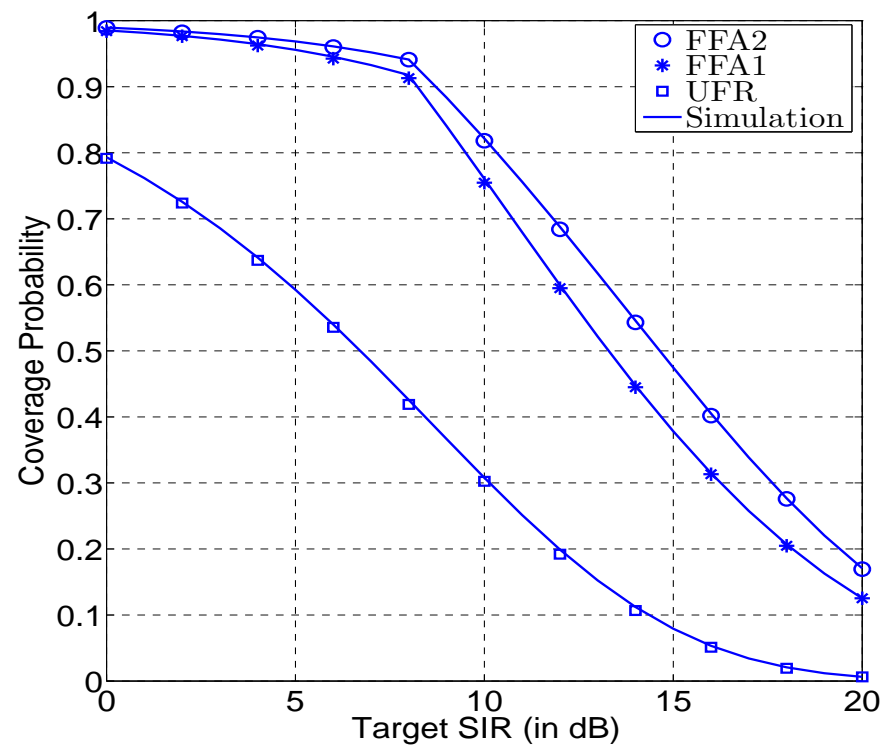

Figure 4. Coverage probability of the D2D link for the proposed schemes relying on FFR. Here we have $\alpha=3, R_{c}=1000 m, P_{c}=46 \mathrm{dBm}$, $P_{d}^{\max }=20 \mathrm{dBm}, S_{d}=8 \mathrm{~dB}, \epsilon=0$.

$S_{d}$, the D2D link is considered to be a LR link and hence will use a new sub-band, which is defined for the LR D2D link according to the proposed frequency allocation schemes. The distance $r$ between the D2D Tx and Rx is also uniformly distributed in $\left[R_{1}, R_{2}\right]$, where the minimum distance $R_{1}$ is set to $30 \mathrm{~m}$ and the maximum distance $R_{2}$ is set to $50 \mathrm{~m}$, i.e. $r \in[30,50] m$, except for Fig. 5. Similar setting for distance between D2D pair has been adopted in [24]-[26]. The power budget of the MBS and D2D links is set to $46 \mathrm{dBm}$ and $20 \mathrm{dBm}$, respectively. We assumed random mapping of the D2D links to the CU's resource blocks and the transmit power of the D2D links is a function of the distance $r$, as defined in Eq. (1) of Section II. The power control factor $\epsilon$ is set to zero for all the results, unless otherwise stated. This means that the D2D links transmit at the maximum power. The transmission channels experience independent Rayleigh fading and a path loss factor of $\alpha=3$. We have analysed the proposed schemes and the benefits of power control in the D2D links by dividing this section into three parts as follows:

\section{A. Fractional Frequency Reuse}

In this section, we analyse the performance of the proposed frequency allocation schemes for the D2D links, when the MBS employs FFR. Explicitly, we evaluate the coverage probability and capacity of the D2D links as well as their impact on the CU's coverage probability. We commence with a discussion of the performance of both FFA1 and FFA2 on the coverage probability of the D2D link, where we consider $\alpha=3$ and $S_{d}=8 \mathrm{~dB}$. Fig. 4 depicts the coverage probability of the D2D link for both FFA1 and FFA2, when the MBS uses FFR against the benchmark scheme of UFR. First of all, it can be clearly seen that the analytical results closely match the simulations. Secondly, UFR results in the lowest coverage probability amongst all the schemes, since the D2D link experiences interference from all the MBSs including the reference $\mathrm{MBS}^{4}$. Interestingly, both the proposed FFA1 and FFA2 schemes provide a significantly better performance than the UFR scheme. The reason for this trend is as follows: the LR D2D links which otherwise have a significantly lower SIR in the UFR scheme, experience no interference from the reference MBS and they experience the same amount of interference as the frequency reuse $\frac{1}{3}$ pattern. Moreover, FFA2 provides a better coverage probability than FFA1, since FFA2 utilizes the cell-edge frequency of the reference cell, whereas FFA1 utilizes the cell-centre frequency of the reference cell. This means that in FFA2 the interference experienced by the SR D2D links is reduced, since it has a frequency reuse of $\frac{1}{3}$, while in FFA1 it obey a frequency reuse 1 pattern, hence increasing the total interference, thereby decreasing the coverage probability for the latter scheme. This can be shown using the analytical expressions as well, when $S_{d} \geq T$. The coverage probability of a typical D2D link, when $S_{d} \geq T$ using FFA1 is given by Eq. (5),

$$
C P_{F F A 1}=\int_{0}^{R_{c}}\left[C P\left(l, S_{d}\right)+\hat{C P}(l, T)\left(1-C P\left(l, S_{d}\right)\right)\right] f_{L}(l) \mathrm{d} l,
$$

where the term $C P\left(l, S_{d}\right)$ denotes the coverage probability of the D2D link, when it relies on the CCR frequency of the reference cell. Similarly, the term $\left[1-C P\left(l, S_{d}\right)\right]$ denotes the coverage probability of the D2D link, when it utilises the CER frequency of the neighbouring cells. Furthermore, the coverage probability of a typical D2D link associated with $S_{d} \geq T$ using FFA2 is given in Eq. (7),

$$
C P_{F F A 2}=\int_{0}^{R_{c}}\left[\tilde{C P}\left(l, S_{d}\right)+\hat{C P}(l, T)\left(1-\tilde{C P}\left(l, S_{d}\right)\right)\right] f_{L}(l) \mathrm{d} l .
$$

Here the term $\tilde{C P}\left(l, S_{d}\right)$ denotes the coverage probability of the D2D link when it utilises the cell-edge frequency of the reference cell. Note that $C P\left(l, S_{d}\right)<\tilde{C P}\left(l, S_{d}\right)$, where $C P\left(l, S_{d}\right)$ and $\tilde{C P}\left(l, S_{d}\right)$ are the coverage probability of the D2D links, when they utilise the CCR frequency and the CER frequency of the reference cell, respectively. In other words, while calculating $\tilde{C P}\left(l, S_{d}\right)$, the interference experienced by the D2D links is reduced, since it has a frequency reuse of $\frac{1}{3}$, while for calculating $C P\left(l, S_{d}\right)$ it obeys unity frequency reuse pattern. In order to compare the coverage probability of

\footnotetext{
${ }^{4}$ Here reference MBS is the one where D2D link is present.
} 


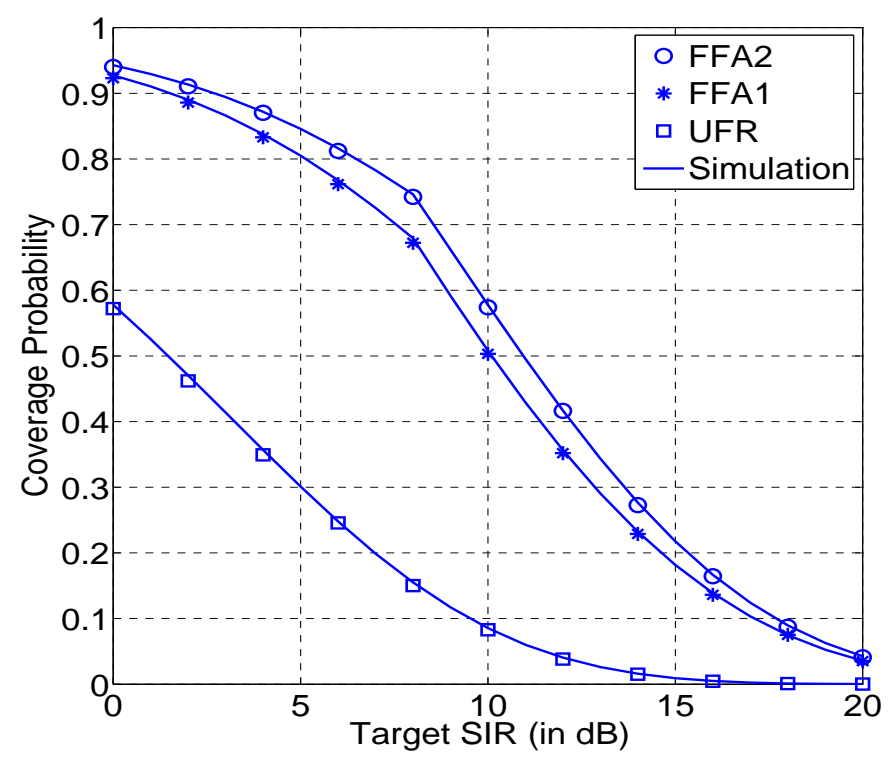

Figure 5. Coverage probability of the D2D link for the proposed schemes relying on FFR. Here we have $\alpha=3, R_{c}=500 m, r \in[20,40], P_{c}=$ $46 \mathrm{dBm}, P_{d}^{\max }=20 \mathrm{dBm}, S_{d}=8 \mathrm{~dB}, \epsilon=0$.

both the schemes, we have rearranged the expressions given in Eq. (5) and Eq. (7) as

$C P_{F F A 1}=\int_{0}^{R_{c}}\left[\hat{C P}(l, T)+C P\left(l, S_{d}\right)(1-\hat{C P}(l, T))\right] f_{L}(l) \mathrm{d} l$,

and

$C P_{F F A 2}=\int_{0}^{R_{c}}\left[\hat{C P}(l, T)+\tilde{C P}\left(l, S_{d}\right)(1-\hat{C P}(l, T))\right] f_{L}(l) \mathrm{d} l$,

Recall that we have $C P\left(l, S_{d}\right)<\tilde{C P}\left(l, S_{d}\right)$ and hence it is apparent from Eq. (16) and Eq. (17) that $C P_{F F A 1}<C P_{F F A 2}$, which conforms with the results presented in Fig. 4.

In Fig. 5, we present coverage probability of D2D links relying on the parameter setting of $R_{c}=500 \mathrm{~m}$ and $r \in$ $[20,40]$, which indicates that our analysis is indeed valid for any parameter setting. Moreover, a change in the value of $R_{c}$ as well as $r$ will not affect the performance trends of the proposed schemes. In other words, both the proposed FFA1 and FFA2 schemes provide a significantly better performance than the UFR scheme. Furthermore, FFA2 provides a better coverage probability than FFA1.

Fig. 6 provides simulation results, where each D2D link has a different target SIR. In particular, we have plotted the coverage probability versus the SIR threshold, where each D2D link has been randomly assigned different target SIRs ranging between $[2,8] \mathrm{dB}$. Interestingly, the behaviour of the proposed schemes investigated in the scenarios, when the D2D links have different target SIRs, remain similar to that of the D2D links having the same target SIR. In other words, both the proposed FFA1 and FFA2 schemes provide a significantly better performance than the UFR scheme.

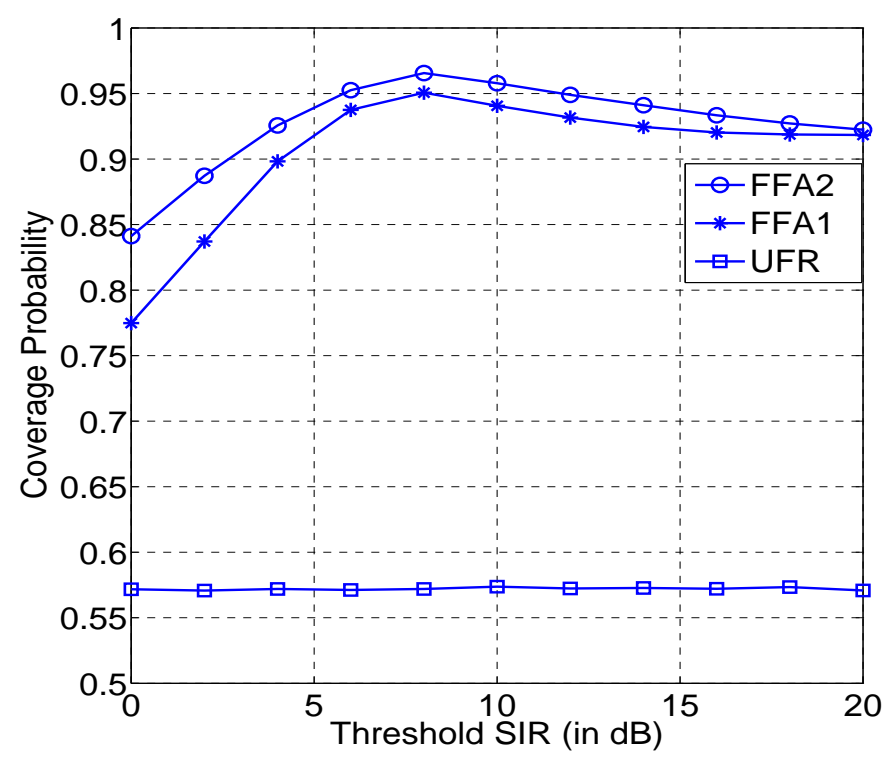

Figure 6. Coverage probability of the D2D link for the proposed schemes relying on FFR. Here we have $\alpha=3, R_{c}=1000 m, P_{c}=46 \mathrm{dBm}$, $P_{d}^{\max }=20 \mathrm{dBm}, \epsilon=0$, and $T \in[2,8] \mathrm{dB}$.

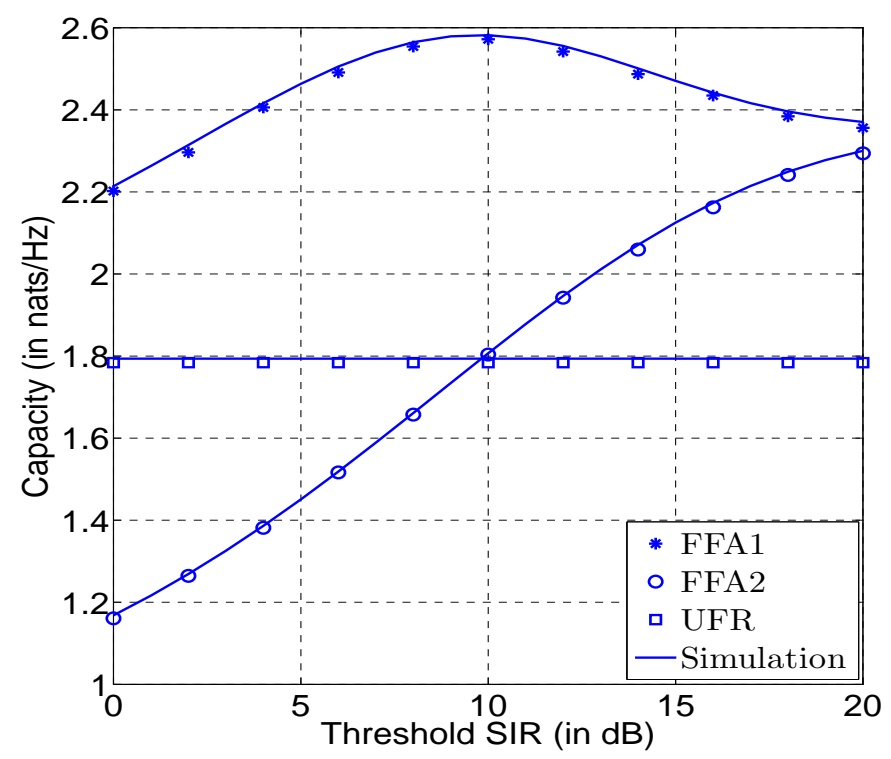

Figure 7. Capacity of the D2D link for the proposed schemes relying on FFR. Here we have $\alpha=3, R_{c}=1000 m, P_{c}=46 \mathrm{dBm}, P_{d}^{\max }=20 \mathrm{dBm}$, $\epsilon=0$.

Moreover, FFA2 provides a better coverage probability than FFA1. We have however not derived the coverage probability and rate expressions, when the D2D links can have different target SIR. It would be interesting to analytically study the performance of D2D links in our future work, when they have different target SIRs.

The capacity of the D2D link for the proposed schemes is shown as a function of the threshold SIR in Fig. 7. It can be observed that as the threshold SIR increases, the capacity of FFA1 first increases and then decreases, but it has 
a significantly higher capacity than the benchmark scheme. A lower value of the threshold SIR may assign too many D2D links from the LR link resources to the SR link resources, while a higher value increases the number of LR D2D links. In either case, the capacity will be reduced. The reason behind this trend is the fact that the capacity of D2D links is influenced both by the SIR of the link as well as by the bandwidth allocated for the frequency resources of the CER and CCR regions in the macrocell. In other words, at a lower threshold SIR, there would be more SR D2D links, which would have a higher bandwidth, but would have a lower SIR, since the interference experienced is increased owing to the employment of the CCR frequency. Hence the overall impact of both these factors would reduce the capacity of D2D links at a low threshold SIR. Similarly, at higher threshold SIRs, there may be more D2D links that are now considered as LR links, that would have provided a higher rate due to a higher SIR, but at the same time it has a lower bandwidth owing to the utilisation of the CER frequency, thereby reducing the overall rate. On the other hand, the capacity of FFA2 increases as the threshold SIR increases. This is due to the fact that in FFA2 D2D links only reuse the CER frequency resources of the MBS. This means that when the threshold SIR increases, the CER frequency resources are increased and hence the capacity of FFA2 increases. Moreover, at a higher threshold SIR, the capacity of FFA2 approaches that of FFA1 due to the fact that both the proposed schemes only use the CER frequency resources at a higher threshold SIR. Note that $S_{d}$ in FFA1 and FFA2 can be chosen according to

$$
C P_{F F A 1, S R}=\int_{0}^{R_{c}} C P_{F F A 1, S R}\left(l, S_{d}\right) \frac{2 l}{R_{c}^{2}} \mathrm{~d} l=\frac{F_{0}}{F}
$$

and

$$
C P_{F F A 2, S R}=\int_{0}^{R_{c}} C P_{F F A 1, S R}\left(l, S_{d}\right) \frac{2 l}{R_{c}^{2}} \mathrm{~d} l=\frac{F_{1}}{F},
$$

respectively. Here $C P_{F F A 1, S R}$ and $C P_{F F A 2, S R}$ denote the specific fraction of D2D links who are categorized into SR links under FFA1 and under FFA2, respectively. Therefore, operators need to carefully choose the value of $F_{i}$ for D2D links aided cellular networks since $S_{d}$ and hence the performance of D2D links depends on $F_{i}$.

We will now analyse the impact of the proposed schemes on the coverage probability of the CUs in Fig. 8 for five different cases: $(i)$ when there are no D2D links and the CU uses UFR, $(i i)$ when there are D2D links and the CU uses UFR, (iii) when the D2D links use FFA1. (iv) when the D2D links use FFA2 $(v)$ when there are no D2D links and the CU uses FFR. All the results are plotted using simulations. It can be observed that the coverage probability of the CU is the lowest when FFA2 is used in the D2D link, since FFA2 only utilizes the CER frequency resources of the MBS. This means that the specific CER users experiencing a low SIR would now experience an even higher interference owing to the presence of D2D links that are reusing the CER user's $\mathrm{RB}$, hence reducing the coverage probability of CER users.

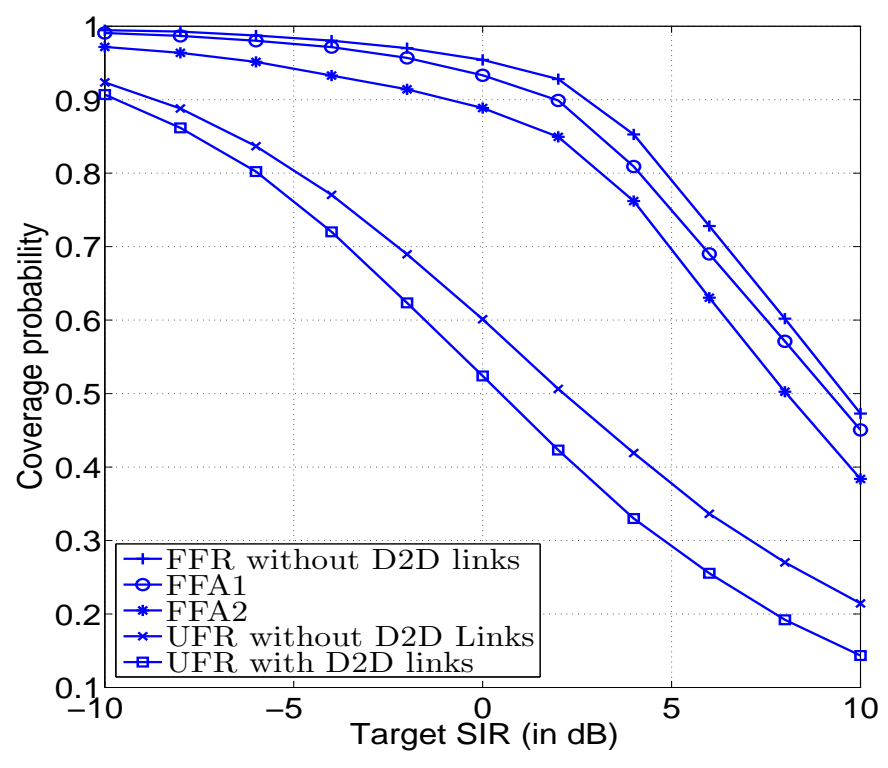

Figure 8. Impact of the proposed FFA1 and FFA2 schemes on the CU's coverage probability. Here $\alpha=3, R_{c}=1000 \mathrm{~m}, P_{c}=46 \mathrm{dBm}$, $P_{d}^{\max }=20 \mathrm{dBm}, S_{C}=3 \mathrm{~dB}, \epsilon=0$.

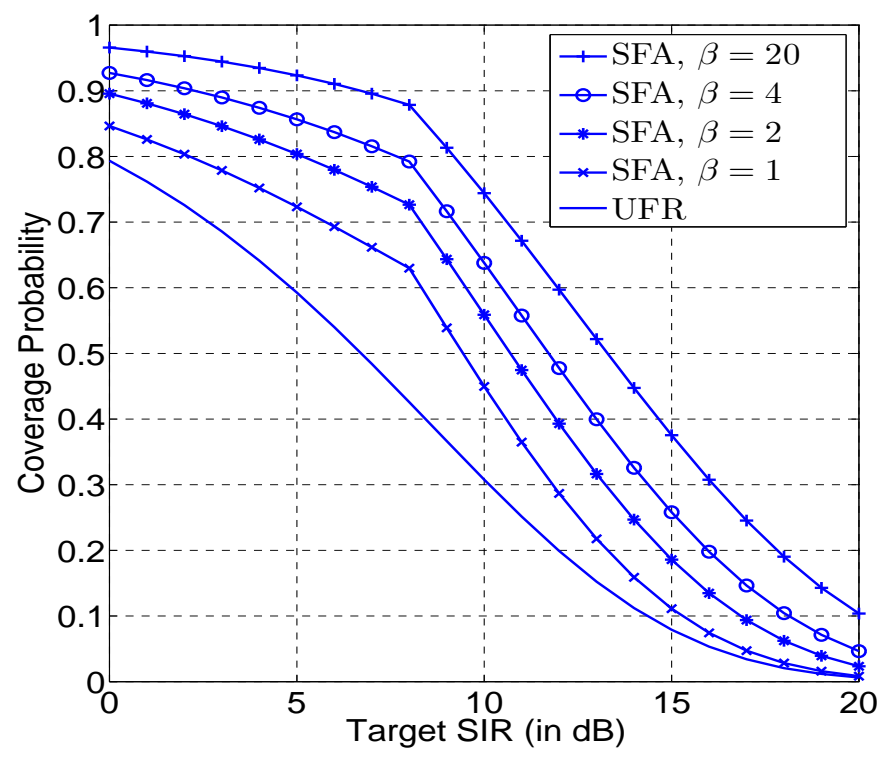

Figure 9. Coverage probability of the D2D link for the proposed SFA scheme. Here we have $\alpha=3, R_{c}=1000 \mathrm{~m}, P_{c}=46 \mathrm{dBm}$, $P_{d}^{\max }=20 \mathrm{dBm}, S_{D}=8 \mathrm{~dB}, \epsilon=0$.

As expected, the coverage probability of CU operating in the absence of D2D links is the highest followed by the CUs when FFA1 is used in the D2D links. It is interesting to note that the coverage probability degradation of $\mathrm{CU}$ is lower, when the D2D links use FFA1 than in the case, when the D2D links use UFR.

\section{B. Soft Frequency Reuse}

In this section, we present the performance analysis of the proposed frequency allocation scheme for D2D links, 
when the macrocell employs SFR, which is also benchmarked against the traditional UFR scheme. We assume that each MBS transmits at a power of $P_{m}\left(=\frac{P_{c}}{\beta}\right)$ in the CCR and at a power of $\beta P_{m}$ in the CER, for ensuring that the maximum power transmitted by any MBS does not exceed its power budget. We would first discuss the coverage probability of D2D links in the case of the SFA scheme for different values of the power control factor $\beta$. It would be fair to compare the UFR scheme to the SFA scheme, when $\beta=1$, since the transmit power of the MBS would be the same for both schemes. It can be clearly seen from Fig. 9 that our proposed scheme has a higher coverage probability than the benchmark scheme. Interestingly, the proposed SFA scheme associated with $\beta=1$ provides a better coverage probability than the benchmark scheme. This improvement in the coverage probability of the SFA scheme over that of the UFR scheme is due to the resultant sub-band diversity gain ${ }^{5}$ achieved by the system, when the D2D link is classified as either an SR or LR D2D link. Moreover, it can be observed that upon increasing the value of the power control factor $\beta$, the coverage probability of D2D links is improved. The reason behind this phenomenon is that as the value of $\beta$ increases, the transmit power $P_{m}$ in the CCR region decreases and hence the interference imposed by the reference MBS on the LR D2D links employing SFA decreases, which results in an increased SIR, hence supporting a higher coverage probability for the D2D links.

We also analysed the capacity of the D2D links using the proposed scheme, when the MBS employs SFR, as shown in Fig. 10. It is interesting to note that the capacity of the proposed scheme increases upon increasing the value of the power control factor $\beta$ of the MBSs. As $\beta$ increases, the D2D links experience a reduced interference, thereby increasing their SIR and hence improving the capacity of the D2D links. Moreover, it can be clearly seen that our proposed scheme performs significantly better than that of the UFR benchmark scheme due to the reduced interference at the $\mathrm{D} 2 \mathrm{D}$ receivers and as a benefit of the sub-band diversity gain achieved by the proposed scheme at the D2D links. However, for the case of $\beta=1$, there is only one reason that is the sub-band diversity gain achieved by the proposed scheme as the transmit power of MBS is same for all the schemes in the curve and hence induce same interference.

\section{Power Control for the D2D links}

In this section, we will analyse the effects of distance-based power control on the D2D links defined in Eq. (1) of Section II using simulations. We will first characterise the impact of the distance $(r)$ on the normalised transmit power of a typical D2D link shown in Fig. 11, parametrized by the power control

${ }^{5}$ A D2D link using the CCR frequency will now be assigned a new sub-band that corresponds to the CER frequency of other cell if its SIR is lower than the threshold SIR $\left(\gamma_{U}>S_{d}\right)$, implying that this D2D link will now experience a new fading power, since the fading is assumed to be independent across the sub-bands. Therefore, there is gain achieved by the system due to allocation of a new sub-band and we call it sub band diversity gain.

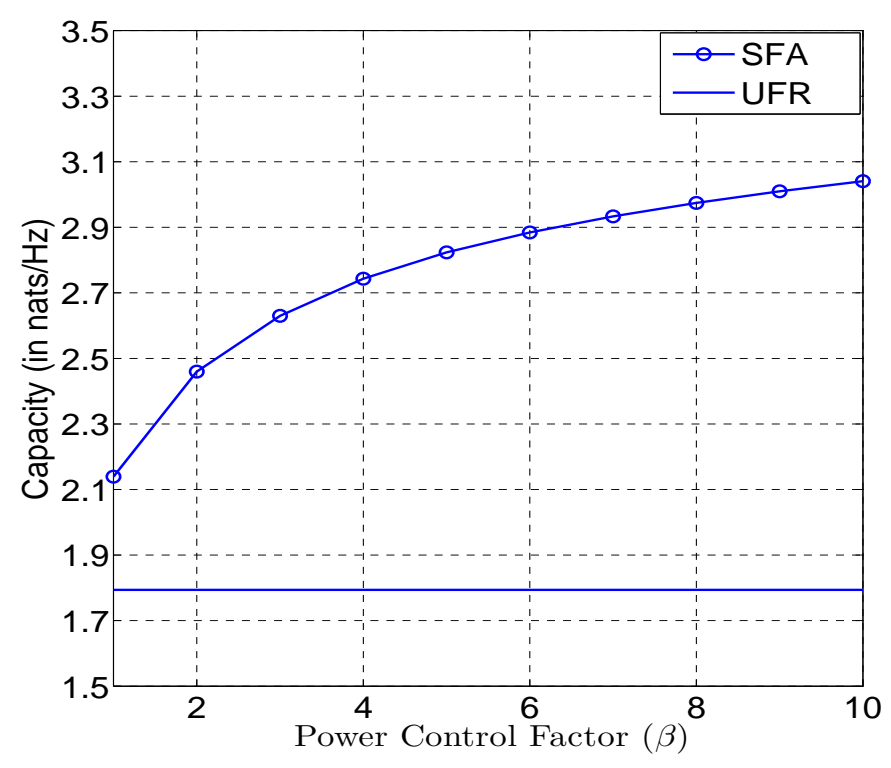

Figure 10. Capacity of the D2D link for the proposed schemes relying on SFR. Here we have $\alpha=3, R_{c}=1000 m, P_{c}=46 \mathrm{dBm}, P_{d}^{\max }=20 \mathrm{dBm}$, $\epsilon=0$.

factor $\epsilon$. It can be clearly seen that as the distance between the D2D transmitter and receiver increases, the transmit power required by the link increases, as expected. It can also be observed that at $\epsilon=0$, all the D2D Txs would transmit at an equal power of $P_{d}^{\max }$ and hence the normalised transmit power would be 1 . However, at $\epsilon=1$, the power received at the D2D Rx would be equal and hence the normalised transmit power is the lowest. Moreover, an interesting observation that can be made from Fig. 11 is that as the power control factor $\epsilon$ increases, the rate at which the transmit power of the D2D Tx decreases is reducing. For example, at a distance of $r=30 \mathrm{~m}$ for the D2D link, as $\epsilon$ varies from 0 to 0.2 , the normalised transmit power is reduced from 1 to 0.66 . However, when $\epsilon$ changes from 0.8 to 1 , the normalized power reduces from 0.195 to 0.13 .

\begin{tabular}{|c|c|c|c|c|}
\hline$\epsilon$ & UFR & FFA1 & FFA2 & SFA \\
\hline 0 & 17.8 & 24.2 & 20.1 & 24.7 \\
\hline 0.5 & 21.0 & 30.0 & 25.5 & 30.4 \\
\hline 1 & 23.8 & 35.0 & 30.6 & 35.2 \\
\hline \multicolumn{5}{|c}{ Table 1 } \\
\hline
\end{tabular}

ENERGY EFFICIENCY OF OUR FOUR FREQUENCY REUSE SCHEMES UPON VARYING THE POWER CONTROL FACTOR $\epsilon$ OF THE D2D LINKS

Table 1 shows the energy efficiency of the different schemes for different power control factors. Energy efficiency is defined as a ratio of the total average rate of D2D links to the total average power of D2D links corresponding to a particular value of the power control factor $\epsilon$ for the different frequency allocation schemes. Therefore, the unit of energy efficiency is nats/s/Watts. Observe that all the proposed schemes provide a better energy efficiency than UFR, whilst FFA1 attains a higher energy efficiency than FFA2, since FFA1 uses a higher bandwidth compared to FFA2. Moreover, SFA achieves a 


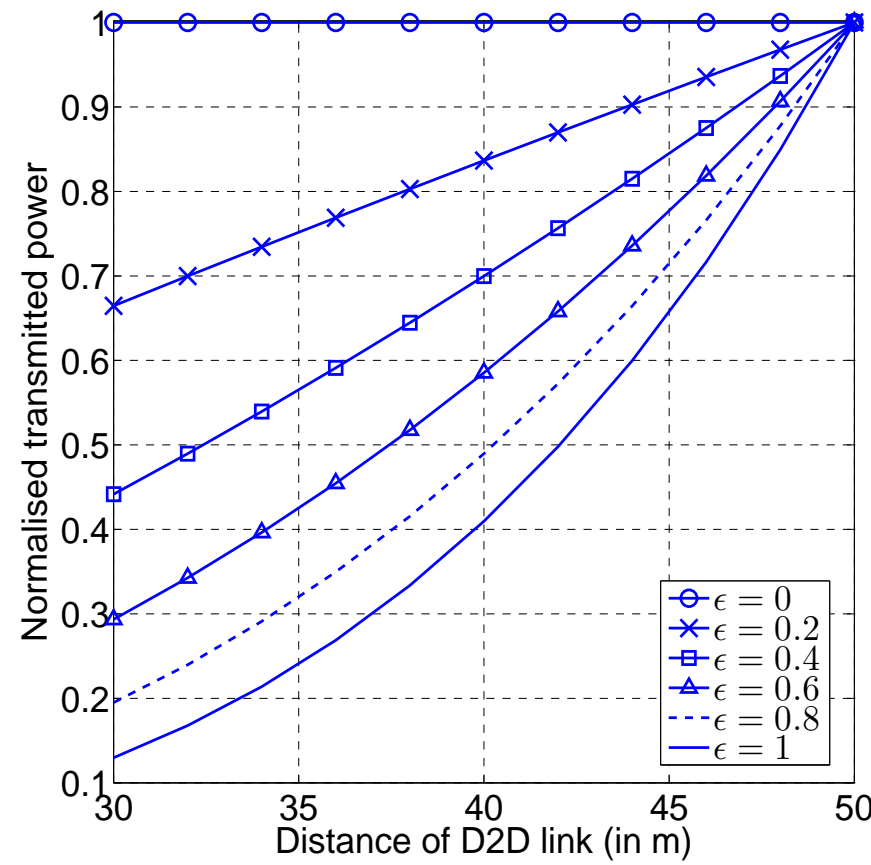

Figure 11. Normalised transmit power versus the D2D link length for different values of the power control factor. Here we have $\alpha=4$.

higher energy efficiency than both FFA1 and FFA2, since it can use all the available bandwidth for D2D communication. Let us now analyse the impact of the power control factor on the energy efficiency. It can be observed that as the power control factor increases, the energy efficiency increases. Interestingly, the increase in energy efficiency is higher, when $\epsilon$ changes from 0 to 0.5 than when it changes from 0.5 to 1. For example, the energy efficiency improvement is $24.3 \%$ when $\epsilon$ changes from 0 to 0.5 , whereas it is only $17.3 \%$, when it changes from 0.5 to 1 for FFA1.

Finally, we characterise the impact of the power control factor $\epsilon$ on the coverage probability of our proposed schemes and of the benchmarker in Fig. 12. It can be clearly seen that as the value of $\epsilon$ increases, i.e. the transmit power of the D2D links decreases, the coverage probability of the D2D links decreases for all the schemes, owing to the reduced SIR of the D2D links. However, the coverage probability reduction of UFR is higher than that for FFA1 and FFA2 in the lower target SIR region. This is due to the fact that the D2D link that has a low SIR due to its high power control factor can be treated as a LR D2D link and thus it will experience a low interference, which in turn would enhance the coverage probability of D2D links in the proposed schemes.

As mentioned previously, the primary motivation of proportional power control of the D2D links is to utilize its energy efficiently. It can be concluded from Fig. 11 and Fig. 12 that when the power control factor is around 0.5, a significant amount of power can be saved at a marginal coverage probability degradation, especially for a low target SIR in case of FFA1 and FFA2. Moreover, Table I suggested
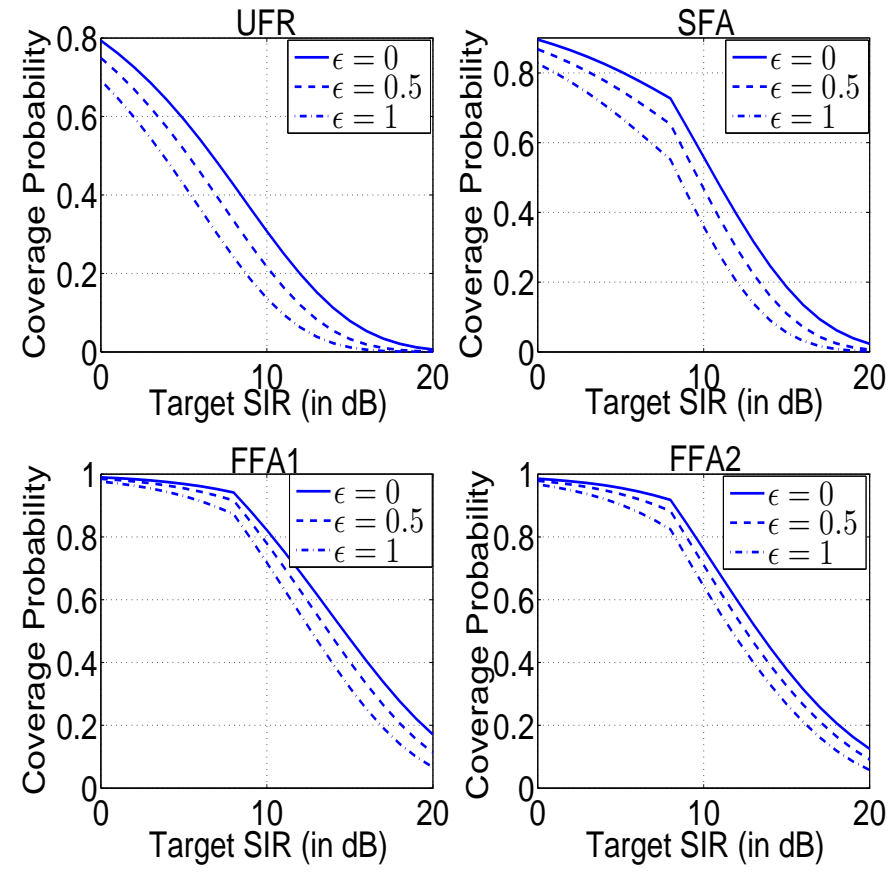

Figure 12. D2D coverage probability versus the target SIR for the proposed schemes parametrized by the power control factor. Here we have $\alpha=3, R_{c}=1000 m, P_{c}=46 \mathrm{dBm}, P_{d}^{\max }=20 \mathrm{dBm}, S_{D}=8 \mathrm{~dB}$,

that 0.5 is a good choice for striking a compromise in terms of energy efficiency at the D2D links for all proposed schemes. Therefore, $\epsilon=0.5$ can be preferred over other values of $\epsilon$.

\section{CONCLUSIONS}

In this treatise, we proposed three frequency allocation schemes, namely FFA1 and FFA2 when MBS uses FFR, while SFA when the MBS employs SFR. These schemes impose less interference on the D2D links, while at the same time satisfying the QoS requirement of the CUs in the macrocell. We analytically derived both the coverage probability and the capacity of D2D links, when they rely on distance-proportional power control under the proposed schemes and found that the simulation results confirm our analysis. It was revealed by our performance results that our frequency allocation schemes significantly outperform the UFR benchmark scheme. Moreover, the impact on the coverage probability of CUs was also studied. Finally, it was shown that the power control factor should be about 0.5 in order to strike an appealing trade-off between the energy consumption and the performance of D2D links. Future work could analyse the performance of the proposed schemes, when the D2D links rely on energy harvesting [27]. It would be also interesting to study the performance of these schemes, when multiple antennas are employed at the D2D transmitter and receiver, when MBS uses ICIC schemes [28]. 


\section{APPENDIX A}

The coverage probability of a D2D link as defined in Section III is given by

$$
\begin{aligned}
P\left[\gamma_{U}(l, r)>T\right] & =P\left[\frac{P_{d}(r) h_{d} r^{-\alpha}}{I_{c d}+I_{d}}>T\right] \\
& =P\left[h_{d}>\frac{T\left(I_{c d}+I_{d}\right)}{P_{d}(r) r^{-\alpha}}\right]
\end{aligned}
$$

Since the fading gain of the D2D link is exponentially distributed, i.e., $h_{d} \sim \exp (1)$, we get,

$$
P\left[\gamma_{U}(l, r)>T\right]=E_{h_{c d, i}, h_{d, j}}\left[\exp \left(-\frac{T\left(I_{c d}+I_{d}\right)}{P_{d}(r) r^{-\alpha}}\right)\right] .
$$

Recall from Eq. (2) that $I_{c d}=\sum_{i \in \phi} P_{c} h_{c d, i} l_{i}^{-\alpha}, I_{d}=$ $\sum_{j \in \psi} P_{d}(r) h_{j} d_{j}^{-\alpha}$ as mentioned in Section II. Since we have Rayleigh channels, $h_{c d, i} \sim \exp (1)$ and $h_{j} \sim \exp (1)$ are considered, hence $I_{c d}+I_{d}$ is the sum of the weighted exponential variates. By exploiting the fact that the weighted exponential variates $h_{c d, i}^{\prime}=w h_{c d, i}$ ( $w$ is the weight of the exponential random variable $h_{c d, i}$ ) can be written as exponential variates associated with a weighted scaling parameter, we have, $h_{c d, i}^{\prime} \sim \exp (w)$. Thus, $I_{c d}+I_{d}$ is the sum of independent and non-identical exponential variates. Let us now use the moment matching technique for evaluating the equivalent distribution of $I_{c d}+I_{d}$, namely that of the total interference experienced by the D2D link. Explicitly the moment matching technique states that the sum of $N$ independent and non-identical Gamma variates $X_{i} \sim \mathcal{G}\left(a_{i}, b_{i}\right)$ can be approximated by a single Gamma variate of $Y=\sum_{i=1}^{N} X_{i} \sim \mathcal{G}(A, B)$, where $A$ and $B$ are defined as

$$
A=\frac{\left(\sum_{i=1}^{N} a_{i} b_{i}\right)^{2}}{\sum_{i=1}^{N} a_{i} b_{i}^{2}} \text { and } B=\frac{\sum_{i=1}^{N} a_{i} b_{i}^{2}}{\sum_{i=1}^{N} a_{i} b_{i}}
$$

Therefore, the distribution of the total interference at the D2D link, obeys $I_{c d}+I_{d} \sim \mathcal{G}(\theta, \lambda)$, where $\theta$ and $\lambda$ are defined based on Eq. (20) and are given by:

$$
\theta(l)=\frac{\left(\sum_{i \in \phi} l_{i}+\sum_{j \in \psi} d_{j}\right)^{2}}{\sum_{i \in \phi} l_{i}^{2}+\sum_{j \in \psi} d_{j}^{2}} \text { and } \lambda(l)=\frac{\sum_{i \in \phi} l_{i}^{2}+\sum_{j \in \psi} d_{j}^{2}}{\sum_{i \in \phi} l_{i}+\sum_{j \in \psi} d_{j}} .
$$

The expression in Eq. (19) can then be simplified and written as,

$$
P\left[\gamma_{U}(l, r)>T\right]=\left(\frac{P_{d}(r)}{\lambda(l) T r^{\alpha}+P_{d}(r)}\right)^{\theta(l)} .
$$

Using the relationship defined in Eq. (1), we can re-write the above expression as:

$$
P\left[\gamma_{U}(l, r)>T\right]=\left(\frac{P_{d}^{\max }}{\lambda(l) T r^{\alpha(1-\epsilon)} R_{2}^{\alpha \epsilon}+P_{d}^{\max }}\right)^{\theta(l)}
$$

Therefore, the coverage probability of a typical D2D link can be formulated as:

$$
\begin{aligned}
C P & =\int_{0}^{R_{c}} \int_{R_{1}}^{R_{2}} P\left[\gamma_{U}(l, r)>T\right] f_{R}(r) \mathrm{d} r f_{L}(l) \mathrm{d} l \\
& =\int_{0}^{R_{c}} \int_{R_{1}}^{R_{2}}\left(\frac{P_{d}^{\max }}{\lambda(l) \operatorname{Tr}^{\alpha(1-\epsilon)} R_{2}^{\alpha \epsilon}+P_{d}^{\max }}\right)^{\theta(l)} \frac{1}{R_{2}-R_{1}} \mathrm{~d} r \frac{2 l}{R_{c}^{2}} \mathrm{~d} l
\end{aligned}
$$

where $f_{R}(r)$ and $f_{L}(l)$ denote the probability density function of $r$ and $l$. In this treatise we assume that the distance $r$ between the D2D Tx and Rx pair is uniformly distributed in $\left(R_{1}, R_{2}\right)$ and these $\mathrm{D} 2 \mathrm{D}$ pairs can be located at a distance $l$ from MBS, which is also distributed uniformly in the cell of radius $R_{c}$. Therefore, we have $f_{R}(r)=\frac{1}{R_{2}-R_{1}}$ and $f_{L}(l)=\frac{2 l}{R_{c}^{2}}$ in Eq. (24). Furthermore, upon solving the inner integral in the above expression given by,

$$
C P(l, T)=\int_{R_{1}}^{R_{2}}\left(\frac{P_{d}^{\max }}{\lambda(l) \operatorname{Tr}^{\alpha(1-\epsilon)} R_{2}^{\alpha \epsilon}+P_{d}^{\max }}\right)^{\theta(l)} \frac{1}{R_{2}-R_{1}} \mathrm{~d} r
$$

we arrive at Eq. (25) given at the top of the next page. Therefore, the coverage probability of D2D links in the UFR scenario can be written as :

$$
C P=\int_{0}^{R_{c}} C P(l, T) \frac{2 l}{R_{c}^{2}} \mathrm{~d} l .
$$

Substituting the value of $C P(l, T)$ from Eq. (25) into Eq. (26), we will obtain $C P$ as given in Eq. (4).

\section{APPENDIX B}

We would first like to derive the coverage probability of the D2D link corresponding to FFA1 by defining it for both the SR and LR D2D links individually.

The coverage probability of the SR D2D link is given by

$$
\begin{aligned}
C P_{F, S R}(l, r) & =P\left[\gamma_{U}(l, r)>T \mid \gamma_{U}(l, r)>S_{d}\right] \\
& =\frac{P\left[\gamma_{U}(l, r)>\max \left\{T, S_{d}\right\}\right]}{P\left[\gamma_{U}(l, r)>S_{d}\right]} .
\end{aligned}
$$

The above conditional probability expression follows from the fact that the SIR of the SR D2D link is higher than $S_{d}$. Similarly, the coverage probability of the LR D2D link is given by

$$
C P_{F, L R}(l, r)=P\left[\hat{\gamma}_{d}^{F}(l, r)>T \mid \gamma_{U}(l, r)<S_{d}\right] .
$$

Note that the LR D2D link reuses the different frequency bands and hence it experiences a new fading power and new interference, which yields a new SIR $\hat{\gamma}_{d}(l, r)$. Since the fading gains are independent of each other, hence $C P_{F, L R}$ can be simplified as

$$
C P_{F, L R}(l, r)=P\left[\hat{\gamma}_{d}^{F}(l, r)>T\right] .
$$




$$
C P(l, T)=\frac{R_{2}}{R_{2}-R_{1}} 2 F_{1}\left[\frac{1}{\alpha-\alpha \epsilon}, \theta(l), 1+\frac{1}{\alpha-\alpha \epsilon}, \frac{-\lambda(l) R_{2}^{\alpha} T}{P_{d}^{m a x}}\right]-\frac{R_{1}}{R_{2}-R_{1}}{ }_{2} F_{1}\left[\frac{1}{\alpha-\alpha \epsilon}, \theta(l), 1+\frac{1}{\alpha-\alpha \epsilon}, \frac{-\lambda(l) R_{1}^{\alpha-\alpha \epsilon} R_{2}^{\alpha \epsilon} T}{P_{d}^{m a x}}\right]
$$

Thus the coverage probability of a D2D link at a distance $l$ from the MBS of the reference cell employing FFA1 is given by

$$
\begin{gathered}
C P_{F F A 1}(l, r)=C P_{F, S R}(l, r) P\left[\gamma_{U}(l, r)>S_{d}\right]+ \\
C P_{F, L R}(l, r) P\left[\gamma_{U}(l, r)<S_{d}\right]
\end{gathered}
$$

Note that $P\left[\gamma_{U}(l, r)>S_{d}\right]$ denotes the percentage of SR D2D links in the macrocell and thus the first term of Eq. (30) gives the coverage probability contribution due to the SR D2D links. Similarly, $P\left[\gamma_{U}(l, r)<S_{d}\right]$ gives the probability of the D2D links being LR links and hence the second term in Eq. (30) defines the contribution of the LR D2D links to the overall coverage probability of D2D links in the reference macrocell. By substituting Eq. (27) and Eq. (29) into Eq. (30), we can reformulate Eq. (30) as :

$$
\begin{array}{r}
C P_{F F A 1}(l, r)=P\left[\gamma_{U}(l, r)>\max \left\{T, S_{d}\right\}\right]+ \\
P\left[\hat{\gamma}_{U}(l, r)>T\right] P\left[\gamma_{U}(l, r)<S_{d}\right] .
\end{array}
$$

Exploring the process used in Appendix A, the coverage probability of a typical D2D link in the network of Fig. 2 using FFA1 is given by

$$
\begin{aligned}
& C P_{F F A 1}=\int_{0}^{R_{c}} C P\left(l, \max \left\{T, S_{d}\right\}\right)+ \\
& \hat{C P}(l, T)\left(1-C P\left(l, S_{d}\right)\right) f_{L}(l) \mathrm{d} l,
\end{aligned}
$$

where $C P\left(l, \max \left\{T, S_{d}\right\}\right)$ and $C P\left(l, S_{d}\right)$ are defined in Eq. (25) and similar to $C P(l, T), \hat{C P}(l, T)$ can be derived for a frequency reuse factor of $\frac{1}{3}$.

\section{APPENDIX C}

The SIR of an SR D2D link that uses $F_{3}$ in an SFR scenario can be written as:

$$
\begin{aligned}
\gamma_{S}(l, r) & =\frac{P_{d}(r) h_{d} r^{-\alpha}}{I_{c d}+I_{d}}, \\
I_{c d} & =\sum_{i \in \varphi} \beta P_{m} h_{c d, i} l_{i}^{-\alpha}+\sum_{i \in \phi \backslash \varphi} P_{m} h_{c d, i} l_{i}^{-\alpha}, \\
I_{d} & =\sum_{j \in \psi} P_{d}(r) h_{j} d_{j}^{-\alpha},
\end{aligned}
$$

where $\varphi=\{0,8,10,12,14,16,18\}$ denotes the specific cells that interfere the desired signal with the power of $\beta P_{m}$, when using $F_{3}$ in their CER. Hence, there is a change in the interference inflicted upon the D2D link in SFR, since the MBS uses power control for cellular communication, which results in a different interference power arriving from each cell. Therefore, corresponding to this definition of SIR, we can define the coverage probability of the SR D2D link in SFA as:

$$
\begin{aligned}
C P_{S, S R}(l, r) & =P\left[\gamma_{S}(l, r)>T \mid \gamma_{S}(l, r)>S_{d}\right] \\
& =\frac{P\left[\gamma_{S}(l, r)>\max \left\{T, S_{d}\right\}\right]}{P\left[\gamma_{S}(l, r)>S_{d}\right]} .
\end{aligned}
$$

By contrast, the coverage probability of the LR D2D links in SFA is given by

$$
\begin{aligned}
C P_{S, L R}(l, r) & \stackrel{(a)}{=} P\left[\hat{\gamma}_{S}(l, r)>T \mid \gamma_{S}(l, r)<S_{d}\right] \\
& \stackrel{(b)}{=} P\left[\hat{\gamma}_{S}(l, r)>T\right] \\
& \stackrel{(c)}{=} P\left[\frac{P_{d}(r) \hat{h}_{d} r^{-\alpha}}{I_{c d}+I_{d}}>T\right]
\end{aligned}
$$

$$
\text { where } \begin{aligned}
I_{c d} & =\sum_{i \in \varphi^{\prime}} \beta P_{m} h_{c d, i} l_{i}^{-\alpha}+\sum_{i \in \phi \backslash \varphi^{\prime}} P_{m} h_{c d, i} l_{i}^{-\alpha} \\
I_{d} & =\sum_{j \in \psi} P_{d}(r) h_{j} d_{j}^{-\alpha} .
\end{aligned}
$$

Here we have $\varphi^{\prime}=\{2,4,6,7,11,15\}$ in (c) of Eq. (35), i.e. the set of macrocells that transmit at a power of $\beta P_{m}$, when using the CER frequency of $F_{1}$. It is important to note that in case of the LR D2D links, the SFA scheme allocates $F_{1}$ and $F_{2}$ to these links and since the channels are independent, the fading experienced is also independent, hence the new SIR is denoted by $\hat{\gamma}_{S}(l, r)$. Referring to Eq. (35), $\hat{\gamma}_{S}(l, r)$ and $\gamma_{S}(l, r)$ correspond to the SIR of the D2D link, when they are using different frequency bands. Thus, we arrive at (b) of Eq. (35) due to the independent fading experienced by the D2D links and hence the corresponding probabilities, i.e. $P\left[\hat{\gamma}_{S}(l, r)>T\right]$ and $P\left[\gamma_{S}(l)<S_{d}\right]$ are independent probabilities. Therefore, similar to the FFR scenario, the coverage probability of the D2D link in the SFA can be obtained as

$$
\begin{aligned}
& C P_{S F A}=\int_{0}^{R_{c}} \int_{R_{1}}^{R_{2}}\left(P\left[\gamma_{S}(l, r)>\max \left\{T, S_{d}\right\}\right]+\right. \\
& P\left[\hat{\gamma}_{S}(l, r)>T\right] P\left[\gamma_{S}(l, r)<S_{d}\right] f_{R}(r) \mathrm{d} r f_{L}(l) \mathrm{d} l .
\end{aligned}
$$

Using the process of Appendix A, the coverage probability of a typical D2D link in the network of Fig. 2 using SFA is given by

$$
\begin{aligned}
& C P_{S F A}=\int_{0}^{R_{c}} C P_{S}\left(l, \max \left\{T, S_{d}\right\}\right)+ \\
& \hat{C P_{S}}(l, T)\left(1-C P_{S}\left(l, S_{d}\right)\right) f_{L}(l) \mathrm{d} l,
\end{aligned}
$$

where similar to $C P(l, T)$ given in Eq. (25), $C P_{S}\left(l, \max \left\{T, S_{d}\right\}\right)$ and $\hat{C P} P_{S}(l, T)$ can be derived for both the CCR frequency and for the CER frequency. 


\section{APPENDIX D}

The capacity of the D2D link employing FFA2 is :

$$
\begin{array}{r}
C_{F F A 2}=\int_{0}^{R_{c}} \int_{R_{1}}^{R_{2}} \frac{1}{3} C_{S}(l, r) P\left[\gamma_{U}(l, r)>S_{d}\right]+ \\
\frac{2}{3} C_{L}(l, r) P\left[\gamma_{U}(l, r)<S_{d}\right] \frac{1}{R_{2}-R_{1}} \mathrm{~d} r \frac{2 l}{R_{c}^{2}} \mathrm{~d} l .
\end{array}
$$

The capacity of the D2D link employing SFA is :

$$
\begin{gathered}
C_{S F A}=\int_{0}^{R_{c}} \int_{R_{1}}^{R_{2}} C_{S}(l, r) P\left[\gamma_{S}(l, r)>S_{d}\right]+ \\
C_{L}(l, r) P\left[\gamma_{S}(l, r)<S_{d}\right] \frac{1}{R_{2}-R_{1}} \mathrm{~d} r \frac{2 l}{R_{c}^{2}} \mathrm{~d} l .
\end{gathered}
$$

\section{REFERENCES}

[1] P. Janis, C. Yu, K. Doppler, C. Ribeiro, C. Wijting, K. Hugl, O. Tirkkonen, and V. Koivunen, "Device-to-device communication underlaying cellular communications systems," in International Journal on Communications, Network and System Sciences, vol. 2, no. 3, June 2009, pp. 169-178.

[2] K. Doppler, M. Rinne, C. Wijting, C. Ribeiro, and K. Hugl, "Deviceto-device communication as an underlay to LTE-advanced networks," IEEE Communications Magazine, vol. 47, no. 12, pp. 42-49, Dec 2009.

[3] W. Oduola, X. Li, L. Qian, and Z. Han, "Power control for deviceto-device communications as an underlay to cellular system," in IEEE International Conference on Communications (ICC), 2014, June 2014, pp. 5257-5262.

[4] X. Zhu, S. Wen, G. Cao, X. Zhang, and D. Yang, "QoS-based resource allocation scheme for device-to-device (D2D) radio underlaying cellular networks," in 19th International Conference on Telecommunications (ICT), 2012, April 2012, pp. 1-6.

[5] J. Hao, H. Zhang, L. Song, and Z. Han, "Graph-based resource allocation for device-to-device communications aided cellular network," in IEEE/CIC International Conference on Communications in China (ICCC), 2014, Oct 2014, pp. 256-260.

[6] D. Zhu, J. Wang, A. Swindlehurst, and C. Zhao, "Downlink resource reuse for device-to-device communications underlaying cellular networks," IEEE Signal Processing Letters, vol. 21, no. 5, pp. 531-534, May 2014.

[7] P. Phunchongharn, E. Hossain, and D. Kim, "Resource allocation for device-to-device communications underlaying LTE-advanced networks," IEEE Wireless Communications, vol. 20, no. 4, pp. 91-100, August 2013.

[8] S. Sun and Y. Shin, "Resource allocation for D2D communication using particle swarm optimization in LTE networks," in International Conference on Information and Communication Technology Convergence (ICTC), 2014, Oct 2014, pp. 371-376.

[9] Z. Xu, G. Li, C. Yang, and X. Zhu, "Throughput and optimal threshold for FFR schemes in OFDMA cellular networks," IEEE Transactions on Wireless Communications, vol. 11, no. 8, pp. 2776-2785, August 2012.

[10] D. Gonzalez, M. Garcia-Lozano, S. Ruiz Boque, and D. S. Lee, "Optimization of soft frequency reuse for irregular LTE macrocellular networks," IEEE Transactions on Wireless Communications, vol. 12, no. 5, pp. 2410-2423, May 2013.

[11] J. Zhang, R. Zhang, G. Li, and L. Hanzo, "Distributed antenna systems in fractional-frequency-reuse-aided cellular networks," IEEE Transactions on Vehicular Technology, vol. 62, no. 3, pp. 1340-1349, March 2013.

[12] S. Kumar, S. Kalyani, and K. Giridhar, "Spectrum Allocation for ICIC-Based Picocell," IEEE Transactions on Vehicular Technology, vol. 64, no. 8, pp. 3494-3504, Aug 2015.

[13] F. Jin, R. Zhang, and L. Hanzo, "Fractional frequency reuse aided twinlayer femtocell networks: analysis, design and optimization," IEEE Transactions on Communications, vol. 61, no. 5, pp. 2074-2085, May 2013.
[14] T. Bansal, K. Sundaresan, S. Rangarajan, and P. Sinha, "R2D2: embracing device-to-device communication in next generation cellular networks," in IEEE Proceedings INFOCOM, 2014, April 2014, pp. $1563-1571$.

[15] W. Wu and Y. Zhang, "Dedicated resource allocation for D2D communications in cellular systems employing FFR," in Sixth International Conference on Wireless Communications and Signal Processing (WCSP), 2014, Oct 2014, pp. 1-6.

[16] H. S. Chae, J. Gu, B.-G. Choi, and M. Chung, "Radio resource allocation scheme for device-to-device communication in cellular networks using fractional frequency reuse," in 17th Asia-Pacific Conference on Communications (APCC), 2011, Oct 2011, pp. 58-62.

[17] H. Kim, J. H. Na, and E. Cho, "Resource allocation policy to avoid interference between cellular and D2D Links/ and D2D links in mobile networks," in International Conference on Information Networking (ICOIN), 2014, Feb 2014, pp. 588-591.

[18] H. Zhu and J. Wang, "Device-to-device communication in cellular networks with fractional frequency reuse," in IEEE International Conference on Communications (ICC), 2014, June 2014, pp. 55035507.

[19] P. Bao, G. Yu, and R. Yin, "Novel frequency reusing scheme for interference mitigation in D2D uplink underlaying networks," in 9th International Wireless Communications and Mobile Computing Conference (IWCMC), 2013, July 2013, pp. 491-496.

[20] D. Feng, L. Lu, Y. Yuan-Wu, G. Li, G. Feng, and S. Li, "Device-to-device communications underlaying cellular networks," IEEE Transactions on Communications, vol. 61, no. 8, pp. 35413551, August 2013.

[21] M. Zulhasnine, C. Huang, and A. Srinivasan, "Efficient resource allocation for device-to-device communication underlaying LTE network," in IEEE 6th International Conference on Wireless and Mobile Computing, Networking and Communications (WiMob), 2010, Oct 2010, pp. 368-375.

[22] T. Novlan, H. Dhillon, and J. Andrews, "Analytical modeling of uplink cellular networks," IEEE Transactions on Wireless Communications, vol. 12, no. 6, pp. 2669-2679, June 2013.

[23] J. Andrews, F. Baccelli, and R. Ganti, "A tractable approach to coverage and rate in cellular networks," IEEE Transactions on Communications, vol. 59, no. 11, pp. 3122-3134, November 2011.

[24] D. Feng, L. Lu, Y. Yuan-Wu, G. Y. Li, G. Feng, and S. Li, "Device-to-device communications underlaying cellular networks," IEEE Transactions on Communications, vol. 61, no. 8, pp. 35413551, August 2013.

[25] X. Lin, J. G. Andrews, A. Ghosh, and R. Ratasuk, "An overview of $3 \mathrm{GPP}$ device-to-device proximity services," IEEE Communications Magazine, vol. 52, no. 4, pp. 40-48, April 2014

[26] D. Feng, L. Lu, Y. Yuan-Wu, G. Y. Li, S. Li, and G. Feng, "Device-todevice communications in cellular networks," IEEE Communications Magazine, vol. 52, no. 4, pp. 49-55, April 2014.

[27] A. H. Sakr and E. Hossain, "Cognitive and Energy Harvesting-Based D2D Communication in Cellular Networks: Stochastic Geometry Modeling and Analysis," IEEE Transactions on Communications, vol. 63, no. 5, pp. 1867-1880, May 2015.

[28] S. Kumar and S. Kalyani, "Impact of Correlated Interferers on Coverage and Rate of FFR and SFR Schemes," IEEE Transactions on Vehicular Technology, vol. 65, no. 1, pp. 434-440, Jan 2016. 


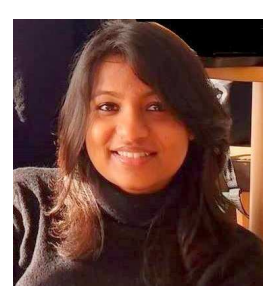

Shruti Gupta received her B.Tech (Hons.) degree in electronics and communication engineering from the LNM Institute of Information Technology (LNMIIT), Jaipur, India, in 2012 and M.Sc. degree (Distinction) in wireless communications from the University of Southampton, UK, in 2013. She is currently pursuing her $\mathrm{PhD}$ degree in the Southampton Wireless (SW) Group, School of Electronics and Computer Science, University of Southampton, UK. Her research interests include cooperative communications and convex optimization. energy harvesting, device-to-device communication,

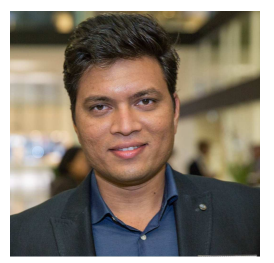

Suman Kumar is an Assistant Professor at the Indian Institute of Technology Ropar (www.iitrpr.ac.in). He has received the B.Tech degree in electronics and communication engineering from the Future Institute of Engineering and Management, Kolkata India in 2010 and the Ph.D. degree in electrical engineering from the Indian Institute of Technology, Madras, India in 2016. He is the recipient of a best paper award at ICWMC2012 held at Venice, Italy. His research interests are broadly in the areas of performance analysis of mobile broadband wireless networks including HetNets, hypergeometric functions, generalized fading models, spectrum sharing, D2D communication.

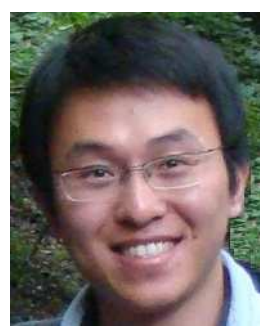

Rong Zhang (M'09) received the B.Sc. degree from Southeast University, China, in 2003, and the $\mathrm{Ph} . \mathrm{D}$. degree from Southampton University, U.K., in 2009. He was an Engineer with China Telecom from 2003 to 2004 and also a Research Assistant with the Mobile Virtual Center of Excellence (MVCE), U.K., from 2006 to 2009. He was a PostDoctoral Researcher with Southampton University from 2009 to 2012. He took an industrial consulting leave from 2012 to 2013 for Huawei Sweden R\&D as a System Algorithms Specialist. Since 2013, he has been an Assistant Professor with the Southampton Wireless Group of ECS, Southampton University. He has authored over $40+$ journals in prestigious publication avenues (e.g., the IEEE and OSA) and many more in major conference proceedings. He serves as a Reviewer of the IEEE TRANSACTIONS/JOURNALS and has served several times as a TPC Member/Invited Session Chair of major conferences. He is a recipient of joint funding from MVCE and EPSRC and is also a Visiting Researcher under the Worldwide University Network.

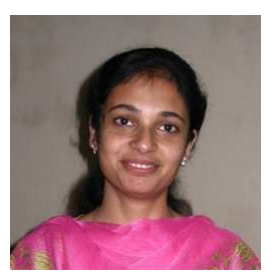

Sheetal Kalyani received the B.E. degree in electronics and communication engineering from the Sardar Patel University, Gujarat India in 2002 and the Ph.D. degree in electrical engineering from the Indian Institute of Technology, Madras, India in 2008. She was a Senior Research Engineer in Centre of Excellence in Wireless Technology, Chennai India from 2008 to 2012. She is currently an Assistant Professor in the Dept. of Electrical Engineering in IIT Madras.

Her current research interests include HetNets, extreme value theory, hypergeometric functions, generalized fading models and statistical learning algorithms for prediction.

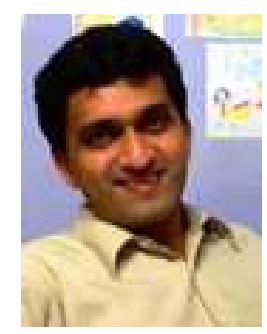

K. Giridhar is a Professor at the Indian Institute of Technology Madras(www.iitm.ac.in), Chennai. He studied BSc (Applied Sciences) at PSG College of Technology, Coimbatore, ME (Electrical Communications) at Indian Institute of Science, Bangalore, and received a $\mathrm{PhD}$ (Electrical Engineering) from University of California, Santa Barbara. Between 1989-90, he was a member of research staff at CRL, Bharat Electronics, Bangalore, and between 1993-94, was a research affiliate in Electrical Engineering at Stanford University, California. Since 1994, he has been with the Department of Electrical Engineering at IIT Madras.

His research interests are broadly in the areas of adaptive signal processing and wireless communications systems, with an emphasis on various transceiver algorithms, custom air-interface design for strategic applications, and performance analysis of mobile broadband wireless networks including HetNets.

Giridhar is a member of the Telecommunications and Computer Networks (TeNeT) Group (www.tenet.res.in) at IITM. He actively collaborates with the Center of Excellence in Wireless Technology (www.cewit.org.in) on MIMO-OFDM broadband access research, resulting in several contributions to IEEE $802.16 \mathrm{~m}$, and currently on proposals to LTE-A and 5G forums.

He serves as a consultant to many Telecom \& VLSI companies in India, and was on a sabbatical in 2004-05 with Beceem Communications. Giridhar has been a visiting faculty at Sri Sathya Sai Institute of Higher Learning, Prasanthi Nilayam, Andhra Pradesh, and at Stanford University, California.

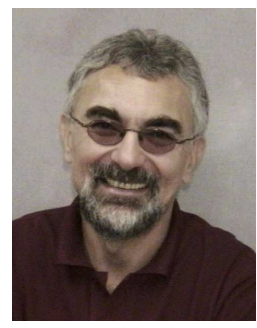

Lajos Hanzo (http://www-mobile.ecs.soton.ac.uk) FREng, FIEEE, FIET, Fellow of EURASIP, DSc received his degree in electronics in 1976 and his doctorate in 1983. In 2009 he was awarded an honorary doctorate by the Technical University of Budapest and in 2015 by the University of Edinburgh. In 2016 he was admitted to the Hungarian Academy of Science. During his 40year career in telecommunications he has held various research and academic posts in Hungary, Germany and the UK. Since 1986 he has been with the School of Electronics and Computer Science, University of Southampton, UK, where he holds the chair in telecommunications. He has successfully supervised about $100 \mathrm{PhD}$ students, co-authored 20 John Wiley/IEEE Press books on mobile radio communications totalling in excess of 10000 pages, published $1600+$ research contributions at IEEE Xplore, acted both as TPC and General Chair of IEEE conferences, presented keynote lectures and has been awarded a number of distinctions. Currently he is directing a 60 -strong academic research team, working on a range of research projects in the field of wireless multimedia communications sponsored by industry, the Engineering and Physical Sciences Research Council (EPSRC) UK, the European Research Council's Advanced Fellow Grant and the Royal Society's Wolfson Research Merit Award. He is an enthusiastic supporter of industrial and academic liaison and he offers a range of industrial courses. $\mathrm{He}$ is also a Governor of the IEEE VTS. During 2008 - 2012 he was the Editor-in-Chief of the IEEE Press and a Chaired Professor also at Tsinghua University, Beijing. For further information on research in progress and associated publications please refer to http://www-mobile.ecs.soton.ac.uk Lajos has $25000+$ citations and an H-index of 60. 